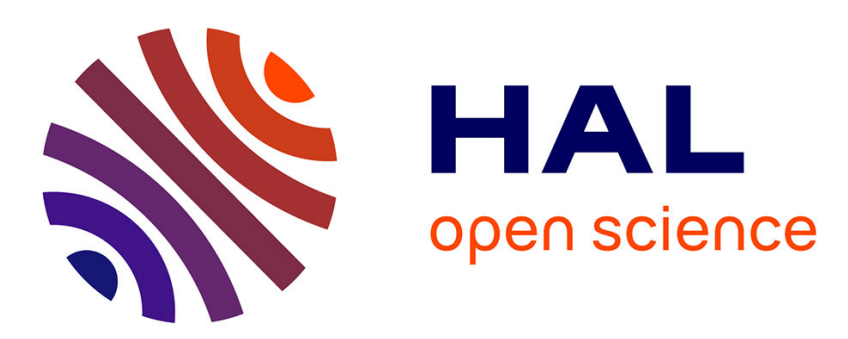

\title{
Sparse and structured decomposition of audio signals on hybrid dictionaries using musical priors
}

Hélène Papadopoulos, Matthieu Kowalski

\section{To cite this version:}

Hélène Papadopoulos, Matthieu Kowalski. Sparse and structured decomposition of audio signals on hybrid dictionaries using musical priors. Journal of the Acoustical Society of America, 2013, 134 (1), pp.666-685. 10.1121/1.4807821 . hal-00823059v2

\section{HAL Id: hal-00823059 \\ https://hal.science/hal-00823059v2}

Submitted on 6 Oct 2013

HAL is a multi-disciplinary open access archive for the deposit and dissemination of scientific research documents, whether they are published or not. The documents may come from teaching and research institutions in France or abroad, or from public or private research centers.
L'archive ouverte pluridisciplinaire HAL, est destinée au dépôt et à la diffusion de documents scientifiques de niveau recherche, publiés ou non, émanant des établissements d'enseignement et de recherche français ou étrangers, des laboratoires publics ou privés. 


\title{
Sparse and structured decomposition of audio signals on hybrid dictionaries using musical priors
}

\author{
Hélène Papadopoulos ${ }^{a)}$ and Matthieu Kowalski \\ Laboratoire des Signaux et Systèmes \\ UMR 8506, CNRS-SUPELEC-Univ Paris-Sud

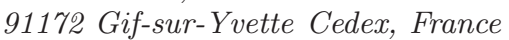

(Dated: September 16, 2013)

\begin{abstract}
This paper investigates the use of musical priors for sparse expansion of audio signals of music, on an overcomplete dual-resolution dictionary taken from the union of two orthonormal bases that can describe both transient and tonal components of a music audio signal. More specifically, chord and metrical structure information are used to build a structured model that takes into account dependencies between coefficients of the decomposition, both for the tonal and for the transient layer. The denoising task application is used to provide a proof of concept of the proposed musical priors. Several configurations of the model are analyzed. Evaluation on monophonic and complex polyphonic excerpts of real music signals shows that the proposed approach provides results whose quality measured by the signal-to-noise ratio is competitive with state-of-the-art approaches, and more coherent with the semantic content of the signal. A detailed analysis of the model in terms of sparsity and in terms of interpretability of the representation is also provided, and shows that the model is capable of giving a relevant and legible representation of Western tonal music audio signals.
\end{abstract}

PACS numbers: 43.75.Xz, 43.75.Zz, 43.60.Hj, 43.60.Pt

\section{I. INTRODUCTION}

We describe in this paper a novel approach for struc- ${ }_{42}$ tured sparse decomposition of a music signal in an over- 43 complete time-frequency hybrid dictionary. Within $\mathrm{a}_{44}$ Bayesian framework, we propose to incorporate musi- 45 cal priors in order to built signal representations that ${ }_{46}$ take into account some "structural" information and that ${ }_{47}$ are more suitable to music than existing methods that ${ }_{48}$ are based on physical signal properties. For this, we ${ }_{49}$ take advantage of the recent work that have been done ${ }_{50}$ on chord estimation and beat tracking in the context ${ }_{51}$ of music content indexing. The model we propose is ${ }_{52}$ inspired from previously proposed Bayesian models for ${ }_{53}$ time-frequency inverse modeling of non-stationary sig- ${ }_{54}$ nals (Wolfe et al., 2004) or sparse linear regression in ${ }_{55}$ unions of bases (Févotte et al., 2008), but presents an es- ${ }_{56}$ sential difference in the way dependencies between coeffi- ${ }_{57}$ cients of the representation are modeled, using the newly ${ }_{58}$ introduced musical priors. One of the goal of this work is ${ }_{59}$ to show that ideas that have emerged in two related but ${ }_{60}$ distinct communities, Sparsity and Music Information ${ }_{61}$ Retrieval, can be exploited jointly to open new perspec- ${ }_{62}$ tive for audio signal processing. Sparse representations ${ }_{63}$ have been used as a basis for extracting high-level in- ${ }_{64}$ formation for MIR applications, such as note extraction ${ }_{65}$ in (Davies and Daudet, 2006), or beat tracking, chord ${ }_{66}$ recognition and musical genre classification in (Ravelli ${ }_{67}$ et al., 2010). Our approach is somewhat different: we ${ }_{68}$ propose to incorporate music content information in or- ${ }_{69}$ der to build structured sparse representations that are ${ }_{70}$ tailored to the analyzed music signal and legible from $\mathrm{a}_{71}$ musical point of view.

a) Author to whom correspondence should be addressed. Electronic mail: helene.papadopoulos@lss.supelec.fr.

\section{A. Structured sparse representation}

The problem of representing an audio signal using a time-frequency dictionary has been given a lot of attention these last few years. The specific problem we consider here is finding an approximation of a music audio signal as a linear combination of elementary waveforms (also called atoms) of a suitably chosen dictionary.

Musical signals are intrinsically structured. A particularity of musical signals is that, very often, several types of components are superimposed such as, among other features, tonal components (the partials of the notes, that are characterized by sinusoids with slowly varying amplitude and frequency) and transients (the attacks of the notes, that correspond to events well-localized in time). This is illustrated in Figure 1 that represents the spectrogram of a glockenspiel signal. The tonals appear as thin horizontal lines whereas the transients appear as sharp vertical lines. These various components may have significantly different behaviors in terms of time-frequency localization. For instance, fast varying transients require short analysis window length, whereas low varying tonals require long windows. Thus, they cannot be optimally represented within the same basis. The Balian-Low theorem (Low, 1985) states that redundancy is necessary for having well-localized functions both in time and frequency for transforms based on local Fourier analysis. This is why hybrid models, allowing a simultaneous representation of different components have been proposed (Hamdy et al., 1996; Verma and Meng, 2000; Daudet and Torrésani, 2002; Molla and Torésani, 2005). These models consider redundant (or overcomplete) dictionaries that are constructed by the concatenation of several families or bases (usually time-frequency atoms, such as Gabor atoms or local cosines; or time-scale atoms, such 


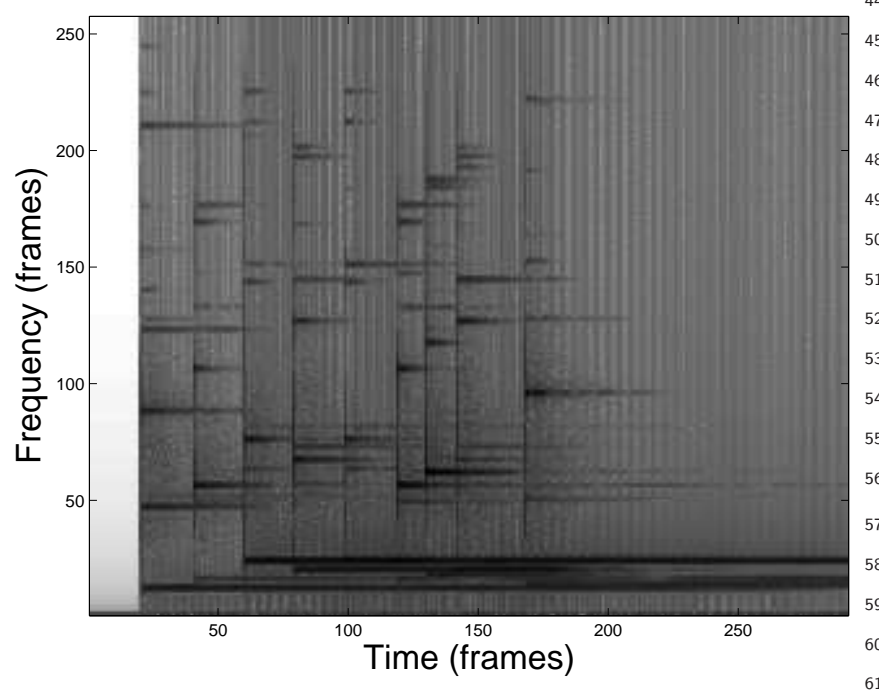

FIG. 1. Time-frequency representation of a recording of a ${ }^{62}$ glockenspiel excerpt. The vertical lines correspond to the at- ${ }^{63}$ tacks of the notes and the horizontal lines correspond to the ${ }^{64}$ partials.

Among the various existing transforms, lapped trans- 68 forms such as the Modified Discrete Cosine Transform 69 (MDCT) (Mallat, 1998; Malvar, 1990) is a standard 70 choice for the bases (Févotte et al., 2006; Kowalski and 71 Torrésani, 2008). This transform is very popular, in par- 72 ticular in high quality audio coding and signal compres- 73 sion applications, because it allows an orthogonal time- 74 frequency transform without blocking effects. Following 75 these approaches, we consider in this work a dictionary 76 built as the union of two MDCT bases with different 77 time-frequency resolutions. The narrow band basis -78 with long time resolution - is used to estimate the tonal 79 parts of the signal, and the wide band basis - with short 80 time resolution - is used to estimate the transient parts. 81 Such a dictionary is overcomplete since the number of el- 82 ements of the dictionary is greater than the length of the 83 signal. The expansion of the signal with respect to the 84 dictionary is thus not unique. Sparsity may be used as a 85 selection criterion for finding the expansion coefficients, 86 in the sense that only a few coefficients of the decompo- 87 sition of the signal on the bases are significantly nonzero. 88 The signal can thus be well approximated by a limited 89 number of coefficients. This problem is often referred to 90 as sparse regression. Sparsity has become a fundamental 91 concept in diverse areas of modern signal processing. It 92 is, for instance, an essential ingredient of popular cod- 93 ing standards such as the MPEG-1 layer III ("MP3") or 94 the MPEG-2 AAC. A review of sparse representations 95 for musical signals and their applications can be found in 96 (Daudet and Torrésani, 2006; Plumbley et al., 2010). 97

A common approach to find a sparse expansion of sig- 98 nals in overcomplete dictionaries consists of minimizing 1 the $\ell_{1}$ norm of the expansion, and is known as basis pur- 2 suit (Chen et al., 1998), or LASSO (Tibshirani, 1996). Other methods include variational approaches (Kowalski, 2009), probabilistic approaches (Kowalski and Torrésani, 2008), greedy methods, such as the Matching Pursuit algorithm (Mallat and Zhang, 1993; Daudet, 2010) and its variants (the Orthogonal Matching Pursuit (Mallat, 1998; Pati et al., 1993), the Molecular Matching Pursuit (Daudet, 2006b)), or Bayesian formulations as for instance EM-based algorithms (Figueiredo, 2003). In the framework of Bayesian variable selection, MCMC (Markov chain Monte Carlo) type approaches have been proposed (Févotte and Godsill, 2006; Févotte et al., 2008). One of the main advantages of the MCMC techniques is their robustness because they scan the whole of the posterior distribution and thus are unlikely to fall into local minima. However, this is done at the expanse of high computational cost.

The concept of structured approximation has been introduced from the observation that significant coefficients are not isolated but tend to form "clusters" in the index space. As mentioned above, audio music signals are well-structured. In the time-frequency plane, the partials of the notes will generate horizontal lines localized in frequency, whereas the attacks of the notes and the percussive sounds will generate vertical lines localized in time. Ideally, this structure should be reflected in the signal decomposition, so that the coefficients have physical interpretability and are more meaningful than isolated coefficients from an analysis perspective. Interpretability is a key concept in sparsity ${ }^{1}$. A signal representation where coefficients can be explained from a theoretical or a physical point of view can help assessing the model accuracy, and provides a suitable representation for higher-level tasks. For instance in music signal analysis, a time-frequency representation where coefficients can be physically interpretable as being part of the tonal layer may be very useful to the multi-f0 estimation task (Yeh et al., 2010). This is why we are interested in finding a signal approximation that is not only sparse, but also structured, by considering dependencies between significant coefficients. Previous approaches that use unstructured priors, such as Bernoulli models have shown that they generate isolated coefficients with high amplitude in both bases (Févotte and Godsill, 2006; Kowalski and Torrésani, 2008). These components do not have any physical or musical meaning and are usually perceived as "musical artifacts" or "musical noise" in the reconstructed signal. Considering dependencies between atoms coefficients and using structured priors allows reducing the number of such undesirable components. Various approaches have been proposed for introducing dependencies between coefficients in the time-frequency domain. These approaches aim at exploiting the fact that significant coefficients tend to be organized into clusters, which results from persistence properties of timefrequency representations. Structures can be modeled directly in the coefficients themselves, such as in (Kowalski, 2009). However, dependencies are often introduced in the time-frequency indices, rather than directly in the coefficients. This results into hierarchical models in which both the coefficients and the addresses of the significant 
Among existing approaches, physical properties resulting in persistency over frequency of the transient layer ${ }_{63}$ can be modeled using structured hierarchical Bernoulli ${ }_{64}$ models on a dictionary built as the union of two MDCT ${ }_{65}^{64}$ bases with different time-frequency resolutions (Kowal- ${ }_{66}$ ski and Torrésani, 2008), binary Markov trees (Crouse ${ }_{67}$ et al., 1998; Molla and Torésani, 2005), or dyadic trees of ${ }_{68}^{67}$ wavelet coefficients used with wavelet bases (Daudet and ${ }_{69}$ Torrésani, 2002); persistency of the tonal layer can be favored using Markov chains, as proposed in (Molla and Torésani, 2005) in the case of a MDCT base; in (Févotte ${ }_{72}$ et al., 2008), structural constraints on the coefficients ${ }_{73}$ that rely on physical properties of the signal are imposed ${ }_{74}$ for both layers. Persistencies of time-frequency coefficients of musical signal are modeled using two types of ${ }_{76}$ Markov chains. It results in a "horizontal structure" for ${ }_{77}$ the tonal layer and a "vertical structure" for the transient ${ }_{78}$ layer.

Enforcing structure between expansion coefficients can ${ }^{80}$ be managed using sequential approaches (Daudet and ${ }^{81}$ Torrésani, 2002; Daudet, 2004; Molla and Torésani, 2005) that first identify the tonal layer using the first basis, and then estimate the transient components from the residual, using the second basis. In (Daudet and Torrésani, ${ }^{83}$ 2002; Daudet, 2004), tonal and transient components are expanded sequentially into local cosine and dyadic ${ }^{84}$ wavelets bases respectively. The method does not rely ${ }^{85}$ on any prior segmentation of the signal. For each layer, ${ }^{86}$ only the largest coefficients in each time frame are re- 87 tained based on threshold values that are estimated adap- 88 tively in a quantization stage. In the framework of audio ${ }^{89}$ coding, (Molla and Torésani, 2005) describes an hybrid 90 model for the expansion of audio signals considering a ${ }^{91}$ redundant dictionary made out of the union of local co- 92 sine and wavelet bases. A recursive scheme is proposed ${ }^{93}$ to estimate the two layers, that relies on the assumption ${ }^{94}$ that the cardinalities of the significance maps have to be ${ }^{95}$ known. A priori estimates for the relative sizes of the 96 tonal and transient layers are obtained based on an algo- 97 rithm that determines local transientness of audio signals 98 (Molla and Torésani, 2004). The approach is used to de- 99 velop an hybrid audio coder that does not rely on prior (time) segmentation of the signal.

As stressed in (Daudet, 2006a), sequential approaches ${ }^{102}$ suffer from two limitations. First, errors in a step are ${ }^{103}$ systematically propagated into the next estimation stage ${ }^{104}$ and thus bias the estimates of the other components. ${ }^{105}$ Second, the choice of a threshold that allows discrimi-106 nating large significant from small residual coefficients ${ }^{107}$ is difficult. An alternative to sequential approaches is ${ }^{108}$ the simultaneous approach of both layers, as proposed in ${ }^{109}$ (Févotte et al., 2008).

Starting from this approach, we build a structured model for sparse signal decomposition within a Bayesian 113 framework. The originality of our work is that we model $_{114}$ dependencies between the expansion coefficients by using 1 priors that are based on musical information.

\section{B. Content-based music information retrieval}

Up to now, additional structure constraints that have been added rely on physical properties of the signal. The recent advances in automatic extraction of content information from audio music signals in the field of Music Information Retrieval (MIR) offers an interesting alternative. Content-based music information retrieval deals with the extraction and processing of meaningful information from musical audio. Techniques developed for searching, retrieving, organizing and interacting in a personalized way with large databases of music signals are often based on the use of musical descriptors that are extracted from the signal, such as the key, the chord progression, the melody or the instrumentation. Musical content information can be used to build structured priors that reflect the content of the signal. For instance, as we propose in this paper, the chord progression provides information about the notes that are present in the signal and can be used to build a prior for the tonal layer. Similarly, the position of the beats is related to the transients and can be used to build a prior for the transient layer. These concepts are introduced in what follows.

\section{Chord estimation}

The chord progression of a piece of music is a very important descriptor because it characterizes its harmonic structure. Here, we want to work directly on audio. The symbolic transcription (the score) of a piece of music is not always available, especially in music genres such as jazz music where there is a large part devoted to improvisation. In addition, algorithms that extract a transcription from an audio signal, such as multi-f0 estimation algorithms (Yeh et al., 2010), are still limited and costly. However, numbers of recent work have shown that it is possible to accurately extract a robust representation of the harmonic content without the use of transcription algorithms. Estimating the chord progression of an audio signal has thus become a very popular task in MIR (Sheh and Ellis, 2003; Bello and Pickens, 2005; Harte and Sandler, 2005; Papadopoulos and Peeters, 2011).

The output of a chord estimation algorithm consists in a progression of chords chosen among a given chord lexicon, that is very often limited to the 24 major and minor triads. Chord estimation on real signals has been favored by the use of the chroma features (Wakefield, 1999) or Pitch Class Profiles (Fujishima, 1999), which are traditionally 12-dimensional vectors, with each dimension corresponding to the intensity associated with one of the 12 semitone pitch classes (chroma) of the Western tonal music scale, regardless of octave. The temporal sequence of chroma vectors over time is known as chromagram. Conceptually, the chromagram is a frequency spectrum folded into a single octave. Pooling the spectrum into twelve bins that correspond to the twelve pitch classes of the equal-tempered scale results in a signal representation that allows identifying pitches by an octave. Each chord may be characterized by the semitone pitch classes 
or chroma that correspond to the notes it is composed 58 of. The use of such a mid-level representation overcomes 59 the problem of automatic transcription.

Various approaches for chroma computation exist. Al- ${ }^{61}$ though they present some variances in the implementa- ${ }^{62}$ tion, they follow in general the same guideline that con- ${ }^{63}$ sists of two main steps:

1. First, a semitone pitch class spectrum (SPS), that ${ }^{66}$ is a log-frequency representation of the spectral ${ }^{67}$ content of the music audio signal, is constructed. ${ }^{68}$ It is expressed in a MIDI-note scale and is in gen- ${ }^{69}$ eral either computed from the Fourier transform or ${ }^{70}$ from the constant-Q transform (Brown, 1991).

2. Secondly, the semitone pitch spectrum is mapped 73 to the chroma vectors. For this, the semitones in 74 octave distance are added up to pitch classes.

The chromagram computation may include some other ${ }_{77}^{76}$ steps such as a pre-processing step that separates har- ${ }_{78}$ monic and noise components, a filtering step that ${ }_{79}$ smoothes the chromagram or a post-processing normal- ${ }_{80}$ ization step that makes the chromagram invariant to dynamics.

We rely in this paper on a chromagram computation method, described in (Papadopoulos and Peeters, 2011), that is based on a constant-Q transform applied on a downsampled signal.

For chord estimation, we rely on the model proposed in 84 (Papadopoulos and Peeters, 2007, 2011), that is based on 85 chord templates and hidden Markov models. We briefly 86 described here the concepts that are used in the rest of 87 the paper. The front-end of our model is based on the 88 extraction of a chromagram that represents the audio 89 signal. The chord progression is then modeled as an 90 ergodic 24-states HMM, each hidden state correspond- 91 ing a chord of a the chord lexicon (CM, .., BM, Cm, 92 $\ldots, \mathrm{Bm})$, and the observations being the chroma vec- 93 tors. The observation chord symbol probabilities are ob- 94 tained by computing the correlation between the obser- 95 vation vectors (the chroma vectors) and a set of chord 96 templates which are the theoretical chroma vectors corre- 97 sponding to the $I=24$ major and minor triads. A state- 98 transition matrix based on musical knowledge (Noland 99 and M., 2006) is used to model the rules from which 100 the transitions between chords result. The chord pro-101 gression over time is estimated in a maximum likelihood102 sense by decoding the underlying sequence of hidden ${ }^{103}$ chords $S=\left(s_{1}, s_{2}, \ldots, s_{N}\right)$ from the sequence of observed ${ }^{104}$ chroma vectors using the Viterbi decoding algorithm.

\section{Beat tracking}

Beat tracking is a challenging problem that has been 1 addressed in a large number of works because beat in- 2 formation is used in many applications in music signal 3 processing, such as music analysis, score alignment or 4 cover version identification. Numerous good overviews 5 on the problem of beat tracking are available, such as 6 for instance (Dixon, 2007; Scheirer, 1998; Klapuri et al., 2006; Davies and Plumbley, 2007). In the present work, we use the beat tracker proposed in (Peeters and $\mathrm{Pa}$ padopoulos, 2011) as a front end of the system. Briefly, this approach aims at simultaneously estimating beat locations with downbeat locations from an audio file. A probabilistic framework in which the time of the beats and their associated beat-positions-inside-a-measure role, hence the downbeats, are considered as hidden states and are estimated simultaneously using signal observations. For this, a reverse Viterbi algorithm that decodes hidden states over beat-numbers is proposed. A beattemplate is used to derive the beat observation probabilities. A machine-learning method, the Linear Discriminant Analysis, allows estimating the most discriminative beat-templates. Two kinds of observations are proposed to derive the beat-position-inside-a-measure observation probability: the variation over time of chroma vectors and the spectral balance. This methods was ranked first for the McKinney Collection test-set during the MIREX 2009 beat tracking contest. We refer the reader to (Peeters and Papadopoulos, 2011) for more details.

\section{Contributions}

Sparse representations of signals have recently proved to be useful for a wide range of applications in signal processing, such as denoising (Févotte et al., 2006), coding and compression (Daudet et al., 2004; Ravelli et al., 2008), source separation (Benaroya et al., 2006; Févotte and Godsill, 2006) or music transcription (Blumensath and Davies, 2004). Here, we focus on the task of denoising an excerpt of musical audio. The approach we propose is in many respects related to previously proposed MCMC schemes for nonlinear approximation in hybrid dictionaries of waveforms. However, a main difference is that we aim at providing a multilayered signal decomposition that fits the music signal, in which the layers can well explain the signal and reflect its music content and can provide more relevant semantic information. By incorporating musical priors, we built a model that is particularly well adapted to music and fits the intrinsic nature of Western tonal music. The denosing application is used as a proof of concept for the description of new musical priors introduced in the paper, and we thus focus on assessing the relevance of the new priors rather than demonstrating the superiority of the method in terms of denoising results (although it is competitive with respect to signal to noise ratio to the state-of-the-art). In this context, we systematically compare our model, in which structural constraints on the coefficients are based on musical prior, to the model proposed (Févotte et al., 2008), and in which structural constraints on the coefficients rely on physical properties of the signal are imposed for both layers, reaching the state-of-the-art in terms of SNR results.

Preliminary results of the proposed approach can be found in (Papadopoulos and Kowalski, 2011). In this article, we propose significant improvements to the signal 
model, in particular by presenting a structured model for the transient layer; we include the result of new experiments; finally we present a detailed analysis of the model and case-study examples.

The remainder of this paper is structured as follows. In Section II, we present our model for sparse signal decomposition on hybrid dictionaries that incorporates musical priors; our main contribution is described in part II.C where we specify our formulation of prior dependence structures in the time-frequency plane. We briefly address the problem of parameters estimation in Section III. In Sections IV and V, we present and discuss the simulation results of our model. Conclusions and perspectives for future work are given in Section VI.

\section{SIGNAL MODEL}

This section introduces first the mathematical model used to represent the audio signal, and then defines the priors chosen in a Bayesian context. Particularly, the new musical priors based on the chromagram and the beat locations are exposed in Section II.C.

\section{A. Model}

In this part, we describe our model for signal decomposition with sparse constraint on a hybrid dictionary of elementary waveforms (Daudet and Torrésani, 2002). The dictionary is constructed as the union of two orthonormal bases with different time-frequency resolution that account respectively for the tonal and the transient parts of the signal. We consider a tree-layer signal model of the form:

$$
\text { signal }=\text { tonals }+ \text { transients }+ \text { residual } .
$$

Let $V=\left\{v_{n}, n=1, \ldots, N\right\}$ and $U=\left\{u_{m}, m=\right.$ $1, \ldots, N\}$ be two MDCT bases of $\mathbb{R}^{N}$ with respectively long frame $\ell_{t o n}$ to achieve good frequency resolution for tonals and short frame $\ell_{\text {tran }}$ to achieve good time resolution for transients. The MDCT is a bijective linear transform and we note $n_{\text {ton }}=\frac{N}{\ell_{\text {ton }}}$ and $n_{\text {tran }}=\frac{N}{\ell_{\text {tran }}}$ the number of frames for each basis (see Figure 2). Here, $n$ and $m$ are time-frequency indexes and will be denoted in the following $n=(q, \nu) \in\left[1, n_{\text {ton }}\right] \times\left[1, \ell_{\text {ton }}\right]_{10}^{9}$ or $m=(q, \nu) \in\left[1, n_{\text {tran }}\right] \times\left[1, \ell_{\text {tran }}\right]$.

The signal is decomposed as a linear combination of ${ }^{11}$ atoms of the two basis $V$ and $U$ that account for the ${ }^{12}$ tonal and transient layers plus a residual part that accounts for the noise and that is not sparse with respect to the two considered bases. We denote $D=V \cup U$ the dictionary made as the union of these two bases. $D$ is ${ }_{14}$ overcomplete in $\mathbb{R}^{N}$, and any $x \in \mathbb{R}^{N}$ admits infinitely many expansions in the form:

$$
x=\sum_{n \in I} \alpha_{n} v_{n}+\sum_{m \in I} \beta_{m} u_{m}+r,
$$

where $I=\{1, \ldots, N\}, \alpha_{n}$ and $\beta_{m}$ are the expansion co- 20 efficients and $r$ represents the noise term.

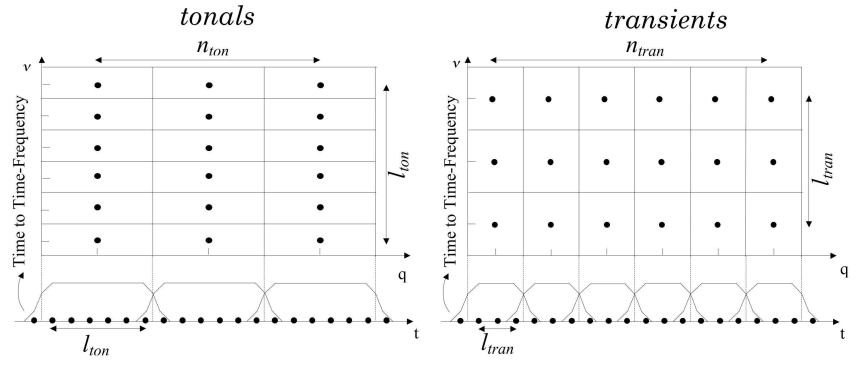

FIG. 2. Illustration of the two MDCT bases that account for the tonal part (long time resolution) and the transient part (short time resolution) of the signal.

We are interested in sparse signals, i.e. signals that may be written as:

$$
x=\sum_{\lambda \in \Lambda} \alpha_{\lambda} v_{\lambda}+\sum_{\delta \in \Delta} \beta_{\delta} u_{\delta}+r
$$

where $\Lambda$ and $\Delta$ are small subsets of the index set $I=$ $\{1, \ldots, N\}$ that account for the significant coefficients, i.e they identify which coefficient of the expansion are significantly non-zero. In what follows, they will be referred to as significance maps.

In order to model sparseness in the time-frequency coefficients, we introduce two indicator random variables corresponding to the significance maps $\Lambda$ and $\Delta, \gamma_{t o n, n}$ and $\gamma_{\text {tran,m }} \in\{0,1\}$ that control the sparsity of the expansion:

$$
\gamma_{t o n, n}=\left\{\begin{array}{ll}
1 & \text { if } n \in \Lambda \\
0 & \text { otherwise }
\end{array} \gamma_{\text {tran }, m}= \begin{cases}1 & \text { if } m \in \Delta \\
0 & \text { otherwise }\end{cases}\right.
$$

We can therefore rewrite Eq. (2) as:

$$
x=\sum_{n \in I} \gamma_{t o n, n} \alpha_{n} v_{n}+\sum_{m \in I} \gamma_{t r a n, m} \beta_{m} u_{m}+r .
$$

The hybrid model is defined by two components: a discrete probability model for the significance maps, and a probability model for the expansion coefficients conditional upon the significance maps. Both of them are described below.

\section{B. Coefficient priors}

The sparseness of the expansion is conceptualized in a hierarchical manner as it is not directly modeled in the coefficients but through the binary indicator random variables $\gamma_{t o n, n}$ and $\gamma_{\text {tran,m }}$ that are attached to each coefficient. As a result, hierarchical priors are given to the coefficients. We assume that, conditional upon the significance maps $\Lambda$ and $\Delta$, the coefficients $\alpha_{n}$ and $\beta_{m}$ 


$$
\begin{aligned}
p\left(\alpha_{n} \mid \gamma_{\text {ton }, n}, \sigma_{\text {ton }, n}\right)= & \left(1-\gamma_{\text {ton }, n}\right) \delta_{0}\left(\alpha_{n}\right)+ \\
& \gamma_{\text {ton }, n} \mathcal{N}\left(\alpha_{n} \mid 0, \sigma_{\text {ton }, n}^{2}\right) \\
p\left(\beta_{m} \mid \gamma_{\text {tran }, m}, \sigma_{\text {tran }, m}\right) & =\left(1-\gamma_{\text {tran }, m}\right) \delta_{0}\left(\beta_{m}\right)+ \\
& \gamma_{\text {tran }, m} \mathcal{N}\left(\beta_{m} \mid 0, \sigma_{\text {tran }, m}^{2}\right)
\end{aligned}
$$

where $\delta_{0}$ is the Dirac delta distribution and, following ${ }_{64}^{63}$ (Wolfe et al., 2004; Févotte et al., 2008), the variances ${ }_{65}$ $\sigma_{\text {ton }, n}$ and $\sigma_{\text {tran }, m}$ are given an inverse-Gamma conjugate ${ }_{66}$ prior distribution:

$$
\begin{gathered}
p\left(\sigma_{\text {ton }, n}^{2} \mid \gamma_{\text {ton }, n}=1, f_{\text {ton }, n}\right)= \\
\mathcal{I} \mathcal{G}\left(\sigma_{\text {ton }, n}^{2} \mid 1, f_{\text {ton }, n}\right) \\
p\left(\sigma_{\text {tran }, m}^{2} \mid \gamma_{\text {tran }, m}=1, f_{\text {tran }, m}\right)= \\
\mathcal{I} \mathcal{G}\left(\sigma_{\text {tran }, m}^{2} \mid 1, f_{\text {tran }, m}\right),
\end{gathered}
$$

where the scale parameters $f_{\text {ton }, n}$ and $f_{\text {tran }, m}$ are para- ${ }^{75}$ metric frequency profiles that aim at taking into account ${ }_{77}$ the decrease of the energy of the signal when the fre- ${ }_{78}$ quency increases (Févotte et al., 2008, Eq. (8)):

$$
f_{\text {ton }, n}=\frac{\lambda_{\text {ton }}}{1+\left(\frac{q-1}{\ell_{\text {ton }} / 3}\right)} \quad f_{\text {tran }, m}=\frac{\lambda_{\text {tran }}}{1+\left(\frac{q-1}{\ell_{\text {tran }} / 3}\right)} .
$$

The parameters $\lambda_{\text {ton }}$ and $\lambda_{\text {tran }}$ are given a non- 84 informative Gamma conjugate prior.

Sparsity is enforced when $\gamma_{\text {ton }, n}=0$ (resp. $\gamma_{\text {tran }, m}=86$ $0)$. In this case, the coefficients $\alpha_{n}$ (resp. $\left.\beta_{m}\right)$ are set to ${ }^{87}$ zero.

\section{Indicator variable priors}

In order to enforce structure between expansion coef- 93 ficients, the significance maps $\Lambda$ and $\Delta$ are given struc- 94 tured priors. We design priors that are tailored to the 95 music signal. The one corresponding to the tonal basis 96 encodes musical information based on harmonic content information using a chord transcription of the analyzed excerpt. The one corresponding to the transient basis is ${ }^{97}$ based on metrical content information using the sequence 98 of beats corresponding to the analyzed excerpt.

To show the relevance of the proposed priors, the ${ }^{100}$ proposed approach will be systematically compared with ${ }^{101}$ a closely related state-of-the art approach described in ${ }^{102}$ Section II.C.3.

In what follows, some results will be illustrated through the representation of the significance maps that are defined by the two binary indicator random variables $\gamma_{t o n, n}$ and $\gamma_{\text {tran,m }}$. In Figure 2, the MDCT bases are illustrated by a tiling of the time-frequency plane, where each tile represents a particular atom. The signif- 2 icance maps in the time-frequency plane are represented 3 through the binary indicator random variable $\gamma$. To 4 each atom corresponds an indicator variable that controls 5 whether this atom is selected $(\gamma=1)$ or not $(\gamma=0)^{2}$.

\section{Model for tonals}

For the significance map corresponding to the tonals, we propose to model dependencies between indicator variables using information about the harmonic content of the audio signal. Ideally, we would assume that we know the score corresponding to the musical excerpt and that, for each time frame $q \in\left\{1, \ldots, n_{t o n}\right\}$, we know which notes the signal is composed of.

However, here, we want to work directly on audio, for which an exact transcription is usually not available. A number of recent work have shown that it is possible to accurately extract robust mid-level representation of the music, such as the chord progression (Papadopoulos and Peeters, 2011), that characterizes its harmonic content.

We propose to give a musical prior to the indicator variables using musical information obtained from a chord progression estimated from the audio file. The output of a chord estimation algorithm consists in a progression of chords chosen among a given chord lexicon that, in general, does not distinguish between any possible combination of simultaneous notes, but is typically reduced to a set of chords of 3 or 4 notes. The number of notes composing the chords will be denoted by $N_{c}$ in the following. Here, we limit our chord lexicon to the 24 major and minor triads $\left(N_{c}=3\right)$. The method we propose could be extended to larger dictionaries.

Each chord is characterized by a set of semitone pitch classes or chroma that correspond to the notes it is composed of. The chord progression does not provide an exact transcription of the music. For instance, passing notes are in general ignored, missing notes in the harmony may be added. Moreover, the chords are estimated regardless of octave. However, experiments show that the provided music content information is sufficient enough to build musically meaningful priors.

We consider two methods for building the structured significance maps for the tonal layer. In the first case, denoted as Method Chord, we use chord information. In the second case, denoted as Method Chroma, the priors are built relying directly on chromagram information.

a. Mapping between MDCT bins and chroma: In order to select atoms of the MDCT base that correspond to the harmonic content of the signal, we first perform a mapping between the MDCT bins and the 12 semitone pitch classes. Given a fixed frame index $q$, let $\left\{p_{\nu}^{M D C T}\right\}_{\nu=1, \ldots, \ell_{\text {ton }}}$ denote the semitone pitch classes corresponding to each frequency MDCT bin.

Assuming a perfect tuning of $A=440 \mathrm{~Hz}$, a MDCT bin of frequency $\nu$ is converted to a chroma $p_{\nu}^{M D C T}$ by the following equation:

$$
p_{\nu}^{M D C T}=\left(12 \log _{2} \frac{\nu}{440}+69\right)(\bmod 12)^{3} .
$$

Note that, a single semitone pitch-class corresponds to several consecutive bins of the MDCT. Because of the logarithmic scale of Western tonal music, the higher the frequency, the larger the number of MDCT bins correspond to a single pitch class. 
b. Method Chords:

Given a fixed frame index $q, 39$ let $\left\{p_{k}^{\text {chord }}\right\}_{k=1, \ldots, N_{c}}$ denote the semitone pitch-classes (chroma) corresponding to the estimated chord $c_{q}$. All bins of the MDCT that correspond to a note belonging to the estimated chord are selected. The indicator variables $\left\{\gamma_{\text {ton },(q, \nu)}\right\}_{\nu=1, \ldots, \ell_{t o n}}$ are given the following membership probabilities:

$$
\begin{aligned}
& P_{\Lambda}\left\{\gamma_{\text {ton },(q, \nu)}=1\right\} \\
& \quad= \begin{cases}p_{\text {ton }} & \text { if } \exists k \in\left[1, N_{c}\right] \mid p_{\nu}^{M D C T}=p_{k}^{\text {chord }} \\
1-p_{\text {ton }} & \text { otherwise },\end{cases}
\end{aligned}
$$

${ }_{14}$ where $0 \leq p_{\text {ton }} \leq 1$. The significance maps corresponding 15 to the tonal layer should reflect the harmonic content of 16 the audio signal. In practice, the value $p_{t o n}$ will be close 17 to 1 (in our experiments, $p_{\text {ton }}=0.9$ ) so that atoms cor18 responding to the notes that are played are given high 19 prior. The significant map for the tonal layer corre20 sponding to the Glockenspiel monophonic audio signal 21 of our test-set is illustrated in Figure 3. A set of atoms 22 is selected at each frame according to the notes of the (note) transcription, regardless of octave. For instance all atoms $\{B 1, B 2, \ldots\}$ corresponding to the semitone B are selected when the first B note of the Glockenspiel is sounded.

The significance maps are given structures of "tubes" that have a musical meaning. Note also that we provide here a "vertical structure" for tonals.
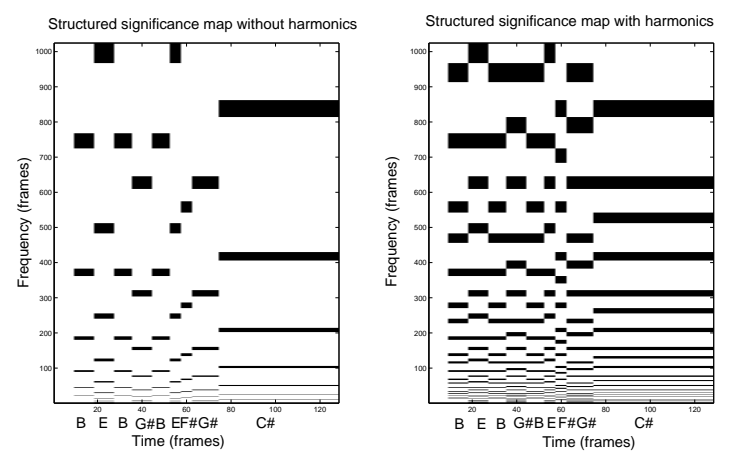

FIG. 3. Structured significance map for tonals for a Glockenspiel excerpt using chord information. Left: only notes composing the chords are considered. Right: higher harmonics are considered. The note transcription is indicated in the bottom.

c. Method Chroma: Given a fixed frame index $q$, let $\left\{a_{k}\right\}_{k=1, \ldots, 12}$ denote the amplitude of each bin ${ }^{48}$ $\left\{p_{k}^{\text {chroma }}\right\}_{k=1, \ldots, 12}$ of the computed chroma vector. Note ${ }^{49}$ that, according to (Papadopoulos and Peeters, 2011), the ${ }^{50}$ chromagram has been normalized so that $\sum_{k=1}^{12} a_{k}=1$. For ${ }^{52}$ each chroma bin, all MDCT bins that correspond to this 1 chroma are selected and given a weighted contribution 2 according to the amplitude in the chroma vector. The 3 indicator variables $\left\{\gamma_{\text {ton },(q, \nu)}\right\}_{\nu=1, \ldots, \ell_{\text {ton }}}$ are given the fol- 4 lowing membership probabilities:

$$
\begin{aligned}
& P_{\Lambda}\left\{\gamma_{\text {ton },(q, \nu)}=1\right\} \\
& \quad=a_{k} \text { if } \exists k \in[1,12] \mid p_{\nu}^{M D C T}=p_{k}^{\text {chroma }}
\end{aligned}
$$
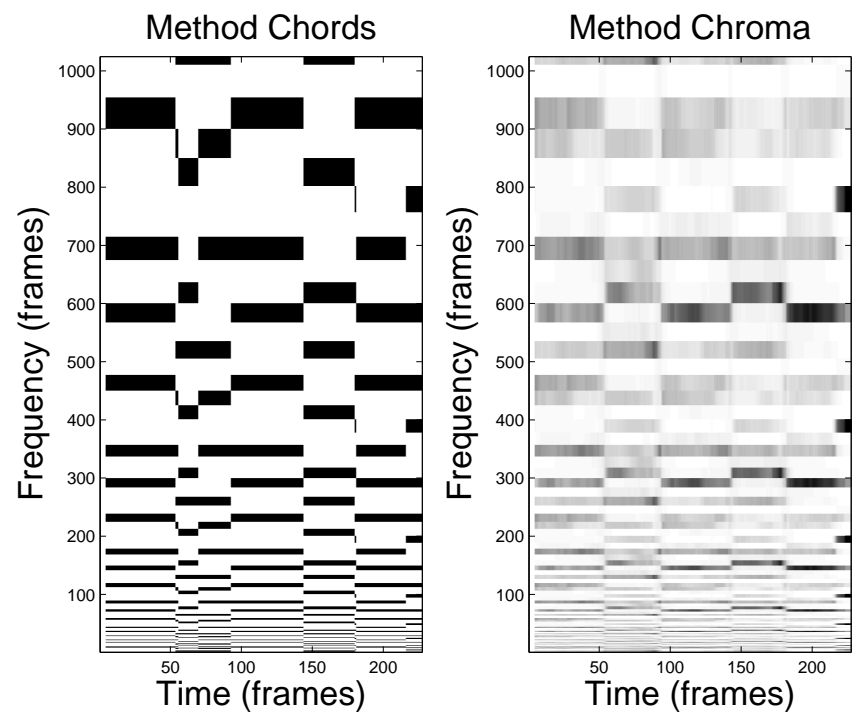

FIG. 4. Structured significance map for tonals for a Beethoven String Quartet Op.127 excerpt using chroma information.

Two additional components may be added to improve the model.

Figure 4 shows the significant maps for the tonal layer corresponding to the Beethoven String Quartet Op.127 audio signal of our test-set obtained with Method Chord [left] and Method Chroma [right].

d. Tuning: The instruments may have been tuned according to a reference pitch different from the standard $A 4=440 \mathrm{~Hz}$. In this case it is necessary to estimate the tuning of the track and Eq. (8) becomes:

$$
p_{\nu}^{M D C T}=\left(12 \log _{2} \frac{\nu}{A_{\text {est }}}+69\right)(\bmod 12)
$$
with the method proposed in (Peeters, 2006).

e. Harmonics: Higher harmonics may be considered in the model. Each note produces a set of harmonics, whose frequencies are whole number multiples of the fundamental frequency ${ }^{4}$, that results in a mixture of non-zero values in the chroma vector corresponding to the chord. For instance a $\mathrm{C} 1$ note will produce the set of harmonics $\{C 1-C 2-G 2-C 3-E 3-G 3-\ldots\}$. They can be considered in the significance maps, as illustrated in the right part of Figure 3. Here we take into account the first 6 harmonics of the notes ${ }^{5}$ 
For the significance map corresponding to the tran- 40 sients, we propose to model dependencies between in- 41 dicator variables using information about the metrical 42 structure of the audio signal. The idea is that, in a piece ${ }_{43}$ of music, most of the transient sounds will occur on beats 44 or beat subdivisions. For instance, drum sounds are gen- 45 erally used to underline the metrical structure (beats, 46 downbeats); in a string quartet piece, bow changes will ${ }_{47}$ generally occur on note changes, which are related to the metrical structure.

The structured prior corresponding to the significance map for tonals is built as follows. The beat positions are estimated from the signal using the beat tracker proposed in (Peeters and Papadopoulos, 2011), described in Section I.B.2. Let $\left\{b_{k}\right\}_{k=1, \ldots, N_{b}}$ denote the $N_{b}$ beat positions (in frames) of the track (subdivisions of beats such as quarter notes or eighth notes can be considered as well). The indicator variables $\left\{\gamma_{\text {tran },(q, \nu)}\right\}_{\nu=1, \ldots, \ell_{\text {tran }}}$ are given the following membership probabilities:

$$
\begin{aligned}
\forall \nu & =1, \ldots, \ell_{\text {tran }} \quad P_{\Delta}\left\{\gamma_{\operatorname{tran},(q, \nu)}=1\right\} \\
& = \begin{cases}p_{\text {tran }} & \text { if } \exists k \in\left[1, N_{b}\right] \mid q=k \\
1-p_{\text {tran }} & \text { otherwise },\end{cases}
\end{aligned}
$$
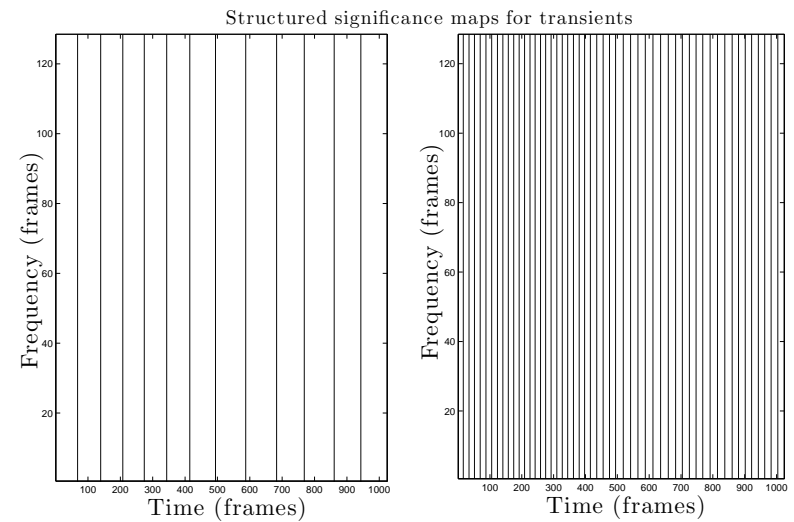

FIG. 5. Structured significance map for transients for a Glockenspiel excerpt using beat location information, resulting in vertical lines in the time-frequency plane. Left: considering 8 beat locations. Right: considering eight-notes locations.
Remark 1 As underlined in (Kowalski and Torrésani, 2008; Févotte et al., 2008), the window lengths for each layer must be significantly different enough to discriminate between tonals and transients. Better results are obtained using a very short window length for the transients $(\approx 3 \mathrm{~ms})$ that is shorter than the duration of a short attack. In this case, several frames may be needed to describe a transient. In practice, we also select vertical lines surrounding the theoretical (or estimated) beat locations in the transient layer prior. Eq. (12) thus becomes:

$$
\begin{aligned}
\forall \nu & =1, \ldots, \ell_{\text {tran }} \quad P_{\Delta}\left\{\gamma_{\text {tran },(q, \nu)}=1\right\} \\
& = \begin{cases}p_{\text {tran }} & \text { if } \exists k \in\left[1, N_{b}\right] \mid q=k \\
p_{\text {tran }} & \text { if } \exists k \in\left[1, N_{b}\right] \mid q=k-1 \\
p_{\text {tran }} & \text { if } \exists k \in\left[1, N_{b}\right] \mid q=k+1 \\
1-p_{\text {tran }} & \text { otherwise. }\end{cases}
\end{aligned}
$$

\section{Baseline approach for comparison}

In this article, we compare our approach, in which structural constraints on the coefficients are based on musical prior, to the baseline model (Févotte et al., 2008), in which structural constraints on the coefficients relying on physical properties of the signal are imposed for both layers. Structure between significant coefficients of the decomposition is introduced to model persistence properties of time-frequency representations of audio signals, so that a horizontal prior structure is given to the indicator variables corresponding to the tonal layer, while vertical prior structure is given to the indicator variables corresponding to the transient layer.

For the tonal layer, persistency in time of the timefrequency coefficients is modeled. Given a fixed frequency index $\nu$, the sequence $\left\{\gamma_{t o n,(q, \nu)}\right\}_{q=1, \ldots, n_{t o n}}$ is modeled by a two-state first-order Markov chain with transition probabilities $P_{t o n, 00}$ and $P_{t o n, 11}$, assumed equal for all frequency indices and initial distribution $\pi_{t o n}$ of each chain taken to be its stationary distribution.

For the transient layer, persistency in frequency of the time-frequency coefficients is modeled. Given a fixed frame index $q$, the sequence $\left\{\gamma_{\text {tran },(q, \nu)}\right\}_{\nu=1, \ldots, \ell_{\text {tran }}}$ is modeled by a two-state first-order Markov chain with probabilities $P_{\text {tran,00 }}$ and $P_{\text {tran,11 }}$, assumed equal for all frames, and with learned initial probability $\pi_{\text {tran }}$.

The tonal and transient models are illustrated in Figure 6. We refer the reader to (Févotte et al., 2008) for more details.

\section{Residual}

The residual signal $r$ is modeled as an independent, identically distributed (i.i.d) Gaussian white noise, with variance $\sigma^{2}$, which is given an inverse-Gamma conjugate prior ${ }^{6}$. 

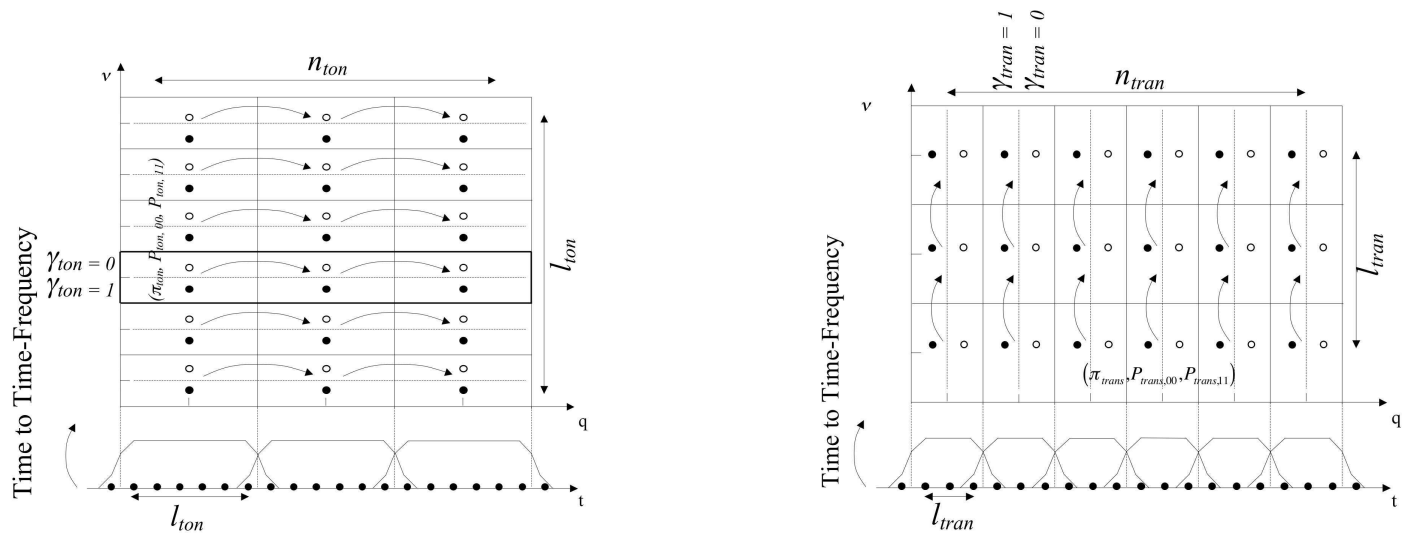

FIG. 6. Structured horizontal model for tonals and vertical model for transients based on time-frequency persistency properties. Adapted from (Févotte et al., 2008).

Let $\theta_{-k}$ denote the set of parameters in $\theta$ except the ${ }^{12}$ parameter $k$. Using Bayes'rule, the conditional distri- ${ }^{13}$ bution of each parameter $k$ conditional upon the other ${ }^{14}$ parameters and the data can be written as:

$$
p\left(k \mid \theta_{-k}, x\right) \propto p(x \mid \theta) p(\theta) .
$$

The conditional distributions are thus proportional to the 19 likelihood of the data times the priors on the parameters. ${ }_{20}$

In order to avoid a nonconvergent Markov chain in the ${ }^{21}$ Gibbs sampler, $\left(\gamma_{\text {ton }}, \alpha\right)$ and $\left(\gamma_{\text {tran }}, \beta\right)$ need to be sam- ${ }^{22}$ pled jointly (Geweke, 1996). As pointed out in (Févotte ${ }^{23}$ et al., 2008), the structure of the dictionary $D=[V U]_{24}$ and the gaussian noise assumption allows alternative 25 block sampling of $\left(\gamma_{t o n}, \alpha\right)$ and $\left(\gamma_{\text {tran }}, \beta\right)$, with the benefit of avoiding any matrix inversion at each iteration of the Gibbs sampler. Indeed, with the gaussian noise assumption, the likelihood of the observations can be written as:

$$
p(x \mid \theta)=\left(2 \pi \sigma^{2}\right)^{-N / 2} \exp \left(-\frac{1}{2 \sigma^{2}}\|x-V \alpha-U \beta\|_{2}^{2}\right) .
$$

Because the Euclidian norm is invariant under rotation, Eq. (15) can be written as:

$$
\begin{aligned}
p(x \mid \theta) & =\left(2 \pi \sigma^{2}\right)^{-N / 2} \exp \left(-\frac{1}{2 \sigma^{2}}\|\underbrace{U^{T}(x-V \alpha)}_{x_{\text {tran } \mid \text { ton }}}-\beta\|_{2}^{2}\right) \\
& =\left(2 \pi \sigma^{2}\right)^{-N / 2} \exp \left(-\frac{1}{2 \sigma^{2}}\|\underbrace{V^{T}(x-U \beta)}_{x_{\text {ton } \mid \text { tran }}}-\alpha\|_{2}^{2}\right) .
\end{aligned}
$$

According to Eq. (16), conditionally upon $\beta$ (resp. $\alpha$ ) and the other parameters, inferring $\alpha$ (resp. $\beta$ ) is thus a simple regression problem with data $x_{\text {ton|tran }}$ (resp. $x_{\text {tran|ton }}$ ), variable $\alpha$ (resp. $\beta$ ) modeled as i.i.d. conditionally upon $\gamma_{t o n}\left(\right.$ resp. $\gamma_{\text {tran }}$ ), and i.i.d. noise, that does not require any matrix inversion.

Briefly, $\left(\gamma_{\text {ton }}, \alpha\right)$ and $\left(\gamma_{\text {tran }}, \beta\right)$ are jointly sampled from by 1) sampling $\gamma_{t o n}$ (resp. $\gamma_{\text {tran }}$ ) from the posterior conditional distribution $p\left(\gamma_{\text {ton, } n} \mid \sigma_{t o n, n}, \sigma, x_{\text {ton } \mid \text { tran }}\right)$ (resp. $\left.p\left(\gamma_{\text {tran, } m} \mid \sigma_{\text {tran, } m}, \sigma, x_{\text {tran } \mid \text { ton }}\right)\right)$, and 2$)$ sampling $\alpha$ (resp. $\beta$ ) from the posterior conditional distribution $p\left(\alpha_{n} \mid \gamma_{\text {ton, } n}, \sigma_{\text {ton }, n}, \sigma, x_{\text {ton } \mid \text { tran }}\right)$ (resp. $\left.p\left(\beta_{m} \mid \gamma_{\text {tran }}, \sigma_{\text {tran, } m}, \sigma, x_{\text {tran } \mid \text { ton }}\right)\right)$. The detailed posterior distributions are given in Appendix A, and we refer the reader to (Févotte et al., 2008; Geweke, 1996) for more details.

The posterior distribution of the other parameters $\sigma_{\text {ton }}, \sigma_{\text {tran }}, \nu_{\text {ton }}$ and $\nu_{\text {tran }}$ are easy to sample from since they have conjugate prior distributions and thus the corresponding posterior will have the same form.

The principal steps of the Gibbs sampler are summarized in Table I, where $K$ is the total number of iterations 
TABLE I. Gibbs sampler steps for parameters inference.

$$
\begin{aligned}
& \text { Initialize } \theta^{(0)} \\
& \text { for } k=1: K+K_{\text {Burnin }} \text { do } \\
& \text { Update tonals } \\
& \text { Update } \gamma_{\text {ton }} \text { and } \alpha \\
& \gamma_{\text {ton }}^{(k)} \sim p\left(\gamma_{\text {ton }} \mid \sigma_{\text {ton }}^{(k-1)}, \sigma^{(k-1)}, x_{\text {ton|tran }}^{(k-1)}\right)(\mathrm{Eq} .(\mathrm{A} 1)) \\
& \alpha^{(k)} \sim p\left(\alpha \mid \gamma_{\text {ton }}^{(k)}, \sigma_{\text {ton }}^{(k-1)}, \sigma^{(k-1)}, x_{\text {ton } \mid \text { tran }}^{(k-1)}\right)(\text { Eq. (A5)) } \\
& \text { Update hyperparameters } \\
& \sigma_{\text {ton }}^{(k)} \sim p\left(\sigma_{\text {ton }} \mid \alpha^{(k)}, \lambda_{\text {ton }}^{(k-1)}\right)(\text { Eq. (A7) }) \\
& \left.\lambda_{\text {ton }}^{(k)} \sim p\left(\lambda_{\text {ton }} \mid \sigma_{\text {ton }}^{(k)}\right) \text { (Eq. (A9) }\right) \\
& \text { Update transients } \\
& \text { Update } \gamma_{\text {tran }} \text { and } \beta \\
& \gamma_{\text {tran }}^{(k)} \sim p\left(\gamma_{\text {tran }} \mid \sigma_{\text {tran }}^{(k-1)}, \sigma^{(k-1)}, x_{\text {tran } \mid \text { ton }}^{(k-1)}\right)(\text { Eq. (A3)) } \\
& \beta^{(k)} \sim p\left(\beta \mid \gamma_{\text {tran }}^{(k)}, \sigma_{\text {tran }}^{(k-1)}, \sigma^{(k-1)}, x_{\text {tran } \mid \text { ton }}^{(k-1)}\right)(\text { Eq. (A6) }) \\
& \text { Update hyperparameters } \\
& \sigma_{\text {tran }}^{(k)} \sim p\left(\sigma_{\text {tran }} \mid \beta^{(k)}, \lambda_{\text {tran }}^{(k-1)}\right)(\text { Eq. (A8)) } \\
& \lambda_{\text {tran }}^{(k)} \sim p\left(\lambda_{\text {tran }} \mid \sigma_{\text {tran }}^{(k)}\right)(\text { Eq. (A10) }) \\
& \text { Update noise } \\
& \sigma^{(k)} \sim p\left(\sigma \mid \alpha^{(k)}, \beta^{(k)}, x\right) \text { (Eq. (A11)) } \\
& \text { end for }
\end{aligned}
$$

The Minimum Mean Square Estimates (MMSE) of the ${ }^{67}$ parameters $\theta$ can then be computed from the Gibbs sam- ${ }^{68}$ ples $\left\{\theta^{\left(K_{\text {Burnin }}\right)}, \theta^{\left(K_{\text {Burnin }}+1\right)}, \ldots, \theta^{(K)}\right\}$ of the posterior ${ }^{69}$ distribution $p(\theta \mid x)$ :
Beethoven String Quartet signal is an excerpt of a string quartet and the Mozart Piano Sonata signal is an excerpt of polyphonic piano music.

TABLE II. Sound excerpts used for evaluation of the model. SR: sampling rate.

\begin{tabular}{|l|c|c|}
\hline Name & SR (Hz) & Duration \\
\hline Glockenspiel & 44100 & $2 \mathrm{~s}$ \\
Misery (Beatles) & 11025 & $11 \mathrm{~s}$ \\
Love Me Do (Beatles) & 11025 & $5 \mathrm{~s}$ \\
Beethoven String Quartet Op.127 - 1 & 11025 & $11 \mathrm{~s}$ \\
Mozart Piano Sonata KV310 -1 & 11025 & $11 \mathrm{~s}$ \\
\hline
\end{tabular}

$$
\begin{aligned}
\hat{\theta}_{M M S E} & =\int \theta p(\theta \mid x) d \theta \\
& \approx \frac{1}{K-K_{\text {Burnin }}} \sum_{k=K_{\text {Burnin }}+1}^{K} \theta^{(k)} .
\end{aligned}
$$

Time-domain source estimates are reconstructed by inverse transform of the estimated coefficients (inverse ${ }^{75}$ MDCT in our case). The denoised estimation is constructed by $\hat{x}=\alpha V+\beta U$.

\section{EXPERIMENTS PROTOCOL AND EVALUATION MEASURES}

In this Section, we describe the test-set and measures 82 we use for the evaluation of the proposed model.

\section{A. Experimental setup}

We present simulation results on 5 musical excerpts 88 of various music styles that are described in Table II. 89 These signals have been chosen because they have diverse 90 characteristics: the Glockenspiel signal is a monophonic 91 signal of a tuned percussion instrument, the Misery and 1 Love Me Do signals are two complex polyphonic excerpts 2 of Beatles songs containing voice and drum sounds, the 3

\section{in}

a. Parameters : The length of the two MDCT bases are set to 1024 samples for the tonal layer and 128 samples for the transient layer, at a sampling rate of $44100 \mathrm{~Hz}$, and respectively to 256 and 32 samples at a sampling rate of $11025 \mathrm{~Hz}$. The MDCT of the clean and noisy signals, with input $S N R=10 d B$ are represented in Figure 7. The MMSE and MAP estimates of the parameters are computed by averaging the last 100 samples of the Gibbs sampler, run for 500 iterations.

We compare a semi-automatic model (denoted as version $S A$ ), assuming that the transcription is known (notes for the monophonic signal, chords for the polyphonic signals, and beat locations) with a fully-automatic model where the music content is directly estimated from the input signal (denoted as version $A$ ). Our approach that incorporates musical priors is compared with the one presented in (Févotte et al., 2008), described in Section II.C.3, in which the priors for both the tonal and transient layers are based on time-frequency persistency properties (version (Févotte et al., 2008)).
\end{abstract}

\section{B. Evaluation measures}

\section{Audio denoising:}

In the context of audio denoising task, artificial noisy signals are created by adding Gaussian white noise to the clean signal with various input SNRs. The case without additional noise $W N$ (without noise) corresponds to a separation into two layers transient + tonal. Partials are expected to be recovered in the tonal layer while attacks or percussive sounds will be recovered in the transient layer. The results in terms of output SNR provide an objective evaluation measure. However, although widely used for assessing algorithm performances, the SNR is not a completely relevant measure of distortion for audio signals and is insufficient to measure the quality or intelligibility of the reconstructed signals. Indeed, it does not reflect exactly the perceived audio quality that includes distortion of the reconstructed signal, musical noise or other artifacts. Subjective evaluation by listening to the signals is also required. Subjective quality assessment of signal reconstruction in source separation or denoising tasks is an active ongoing topic of re- 

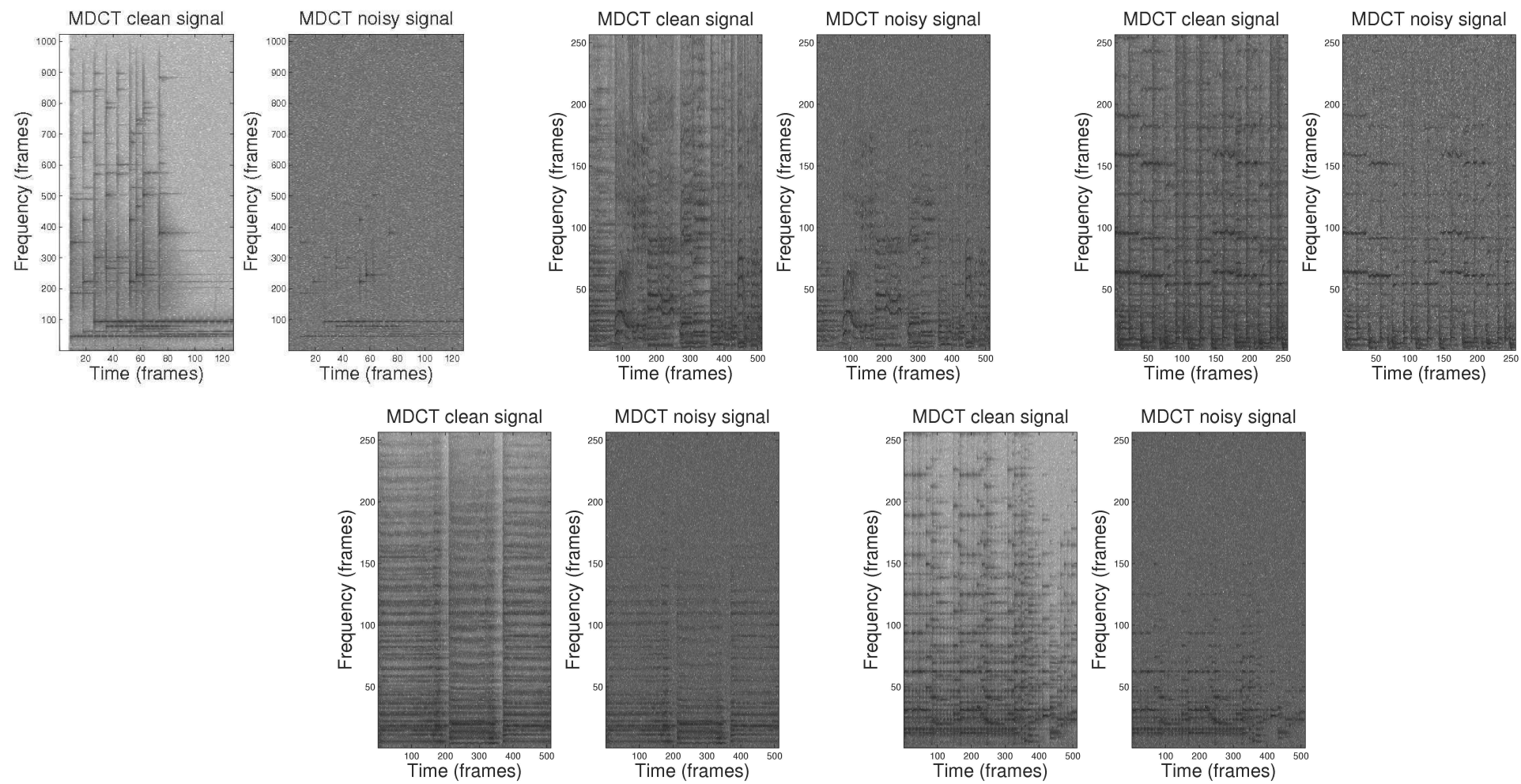

FIG. 7. MDCT of the clean and noisy signals, with input $S N R=10 d B$. Form top top bottom : Glockenspiel, Misery, Love Me Do, Beethoven and Mozart excerpts.

search (Vincent et al., 2006; Emiya et al., 2010; Rohden- 30 burg et al., 2005). It has been found that statistically 31 significant results can be obtained from listening tests ${ }_{22}$ with less than ten non-expert subjects (Vincent et al., 33 2006). However, conducting large-scale listening tests is out of scope of this work, and the subjective assessment of the results is limited here to an analysis and description by the authors of the audio excerpts obtained by the proposed algorithm. All the audio excerpts are available at http://webpages.Iss.supelec.fr/perso/ ${ }^{34}$ matthieu.kowalski/jasa/jasa.htm (date last viewed ${ }_{36}^{35}$ $01 / 20 / 13)$

\section{Sparsity:}

In this work, we aim at obtaining a dual representa- ${ }^{2}$ tion of the signal that is sparse, but that is also struc- ${ }^{43}$ tured and is meaningful according to the music content of the analyzed audio excerpt. A number of criteria for measuring the sparsity of an expansion have been pro- 44 posed. Among them, Rényi entropies (Rényi, 1961), a generalization of the Shannon entropy, have been intro- 45 duced in (Baraniuk et al., 2001) as measures for estimat- 46 ing the complexity and information content of a signal 47 through its time-frequency representation. It has been ${ }_{48}$ shown that minimizing the complexity or information of 49 a time-frequency representation of a signal is equivalent 50 to maximizing its concentration, peakiness, and resolu- 51 tion. Let $\Phi \in \mathcal{L}^{2}\left(\mathbb{R}^{2}\right)$ be a time-frequency representation of a unit-energy signal $s \in \mathcal{L}^{2}(\mathbb{R})$ (for instance, in this article $\Phi$ is the MDCT transform of the signal). The Rényi entropies of $\Phi$, defined as:

$$
R_{\alpha}\left(\Phi(t, f)_{s}\right)=\frac{1}{1-\alpha} \log _{2} \iint \Phi(t, f)_{s}^{\alpha} d t d f, \alpha \in[0,1],
$$

may be interpreted as sparsity measures and have thus been used as a criterion for obtaining a sparse expansion of an audio signal (Jaillet and Torrésani, 2004; Liuni et al., 2011). In the present work, we use a Rényi entropy criteria as an evaluation measure to compare the sparsity of the significance maps. We present here results with $\alpha=0.9$, similar results were obtained with different criteria.

Sparsity is also measured in terms of the percentage of atoms selected in the significance maps.

\section{Computational performances}

The algorithms are implemented in MATLAB and performed on a MacBook Pro Intel Core 2 Duo clocked at $2.4 \mathrm{GHz}$ with $2 \mathrm{~GB}$ RAM. The computation time of the proposed method is similar to the one obtained with (Févotte et al., 2008), $\approx 270 \mathrm{~s}$ for processing the Glockenspiel signal for instance. Note that the use of MCMC schemes generates high computational costs. 
The aim of this section is to provide an analysis of the 60 proposed approach for sparse and structured expansion 61 of audio signals on overcomplete dictionaries. In order 62 to compare our approach to previous work, we evaluate 63 the performance of the proposed approach for the task 64 of audio denoising. Our purpose is to show how expert 65 music knowledge can be used to build priors to obtain a 66 relevant signal decomposition. The main contribution of 67 the article is the design of new musical priors. In order 68 to evaluate the impact of the prior itself, we compare our results with (Févotte et al., 2008), that specifically differs from the proposed method in the priors. The impact 69 of the various parameters (tuning, harmonics, and priors settings) is studied. We also provide a detailed analysis 70 of our model in terms of sparsity and in terms of interpretability of the representation. We wish to show that 71 the use of priors built on music content information al- 72 lows making the structure of the signal legible and leads 73 to a representation that has a musical/physical meaning. 74

\section{A. Structured representation} the case of the Beethoven excerpt, with $S N R_{i n}=10 \mathrm{~dB}$. The chords (especially those on beats 2 and 3 ) sound more "round", and the notes are better held. It should be noticed that listening tests in general do not reveal noticeable improvement when using musical prior also for the transient layer, even though the reconstructed signal in the case of the Mozart excerpt with $S N R_{\text {in }}=$ 0db sounds a little "flatter" without musical prior for the transient layer.

\section{B. Semi-automatic versus automatic approach}

\section{Tonal layer}

Table III shows that the SNR results obtained with an automatic approach $(A)$, where the tonal content is directly estimated from the audio (using a chord estimation algorithm), are similar to the ones obtained with a semiautomatic approach $(S A)$, where the transcription (chord ground-truth) is given as an input of the model. Results are similar at the same time in terms of SNR, in terms of legibility of the significance maps, and in terms of listening tests. The results of chord estimation are indicated in Table III and show that the chord estimation is not perfect. Two scores are considered: Exact Estimation $E E$ corresponds to the rate of chords correctly detected; Exact + Neighbor $E+N$ corresponds to the rate of correctly detected chords including neighboring chords ${ }^{7}$. It can be seen that the exact chord estimation results may be low, especially in low input SNR conditions (for instance $E E=28.35 \%$ for the Love Me Do excerpt, with $S N R_{\text {in }}=0$. However, most errors correspond to neighboring chords (high $E+N$ results), which indicates that most of the notes present in the signal are correctly taken into account. A rough estimation of the tonal content is thus sufficient to built relevant prior for the significance map corresponding to the tonal layer.

\section{Transient layer}

The difference between the SNR results obtained with an automatic approach, where the beats are directly estimated from the audio (using a beat tracking algorithm) and a semi-automatic approach where the beat locations are given as an input of the model, was in each case $\leq 0.1 \mathrm{~dB}$ (For conciseness, detailed results are not reported here). Here again, the transcription does not need to be perfect to build relevant prior for the significance map corresponding to the transient layer.

\section{Influence of musical priors for the transient layer only}

Table IV shows the effect of using musical priors for the transient layer only (and using frequency persistencybased priors for the tonal layer, as in (Févotte et al., 2008)), denoted as case Method tr. In terms of SNR, 


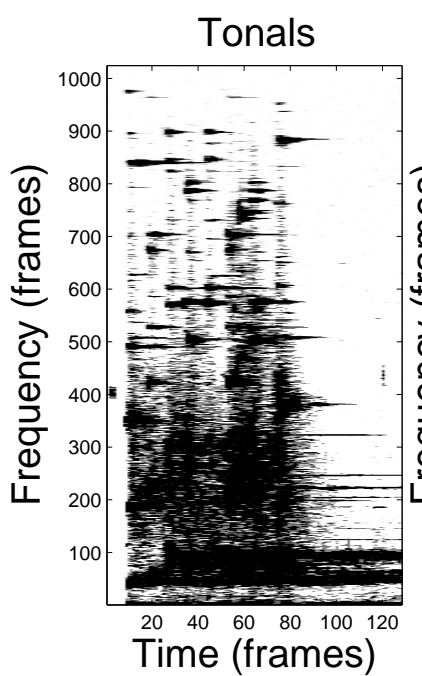

Tonals

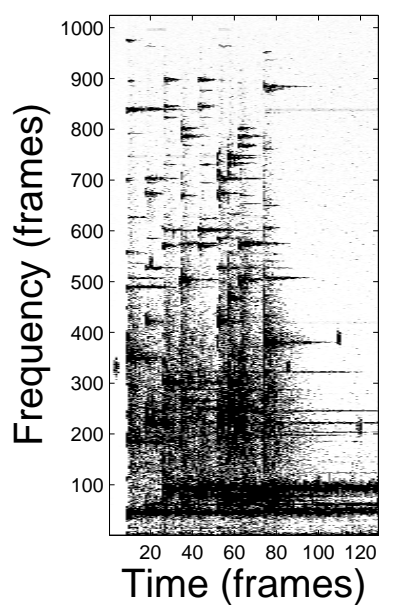

Transients

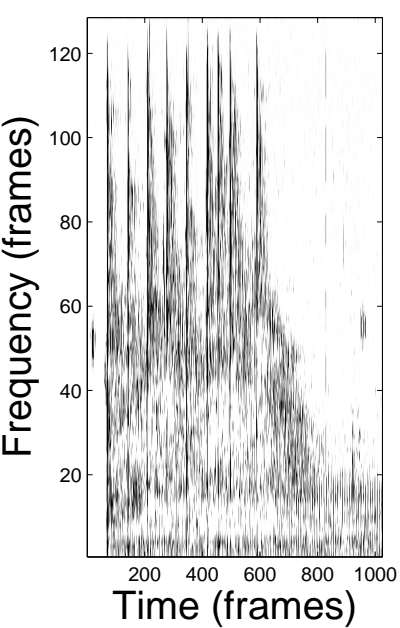

Transients

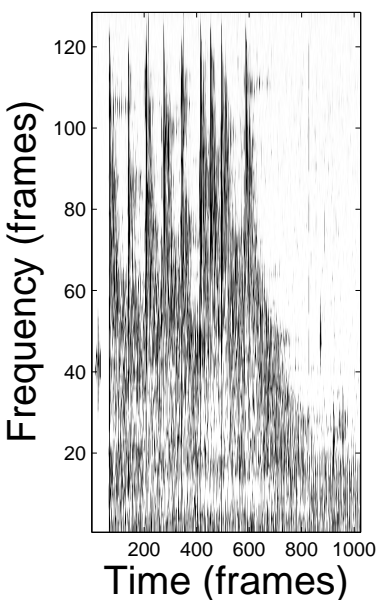

Tonals

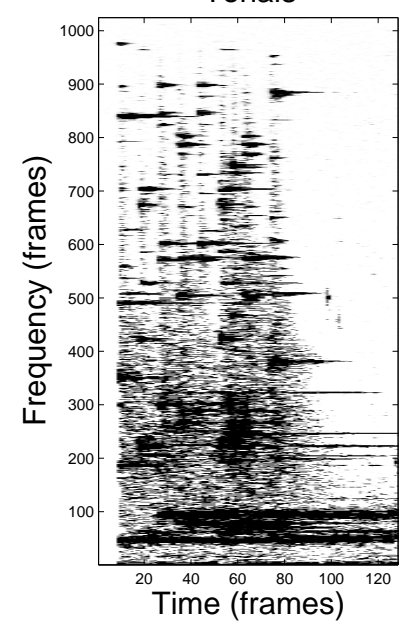

Tonals

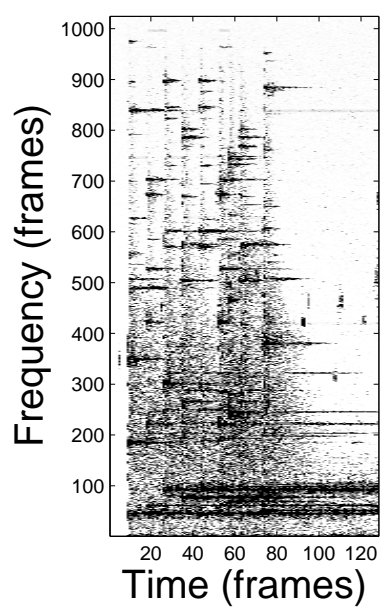

Transients

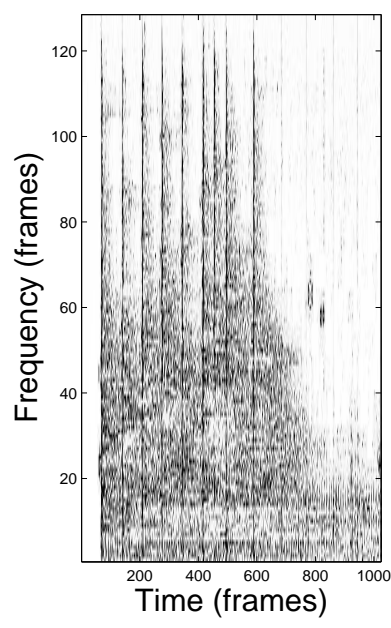

Transients

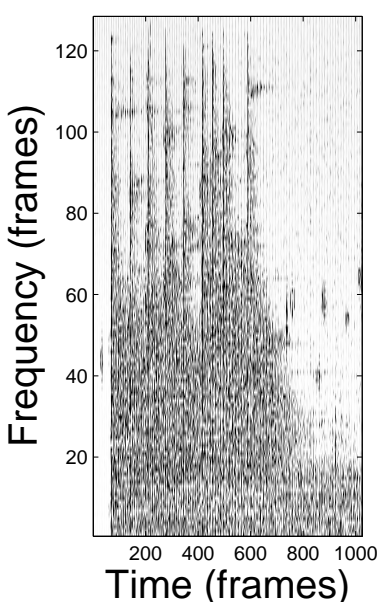

FIG. 8. Significance maps of the tonal and transient bases (MMSE estimates) for the Glockenspiel excerpt, case $W N$. Left-top: approach (Févotte et al., 2008); Left-bottom: proposed approach using musical priors for the transient layer only; Right-top: proposed approach using musical priors for the tonal layer; Right-bottom: proposed approach using musical priors for both the tonal and transient layers.

musical priors for the transient layer yield results that 8 are equivalent to state-of-the-art results, except for poly- 9 phonic clean signals. For monophonic and polyphonic 10 noisy signals, the use of musical priors for transients results in a tonal layer that may be more consistent with the music content: the partials of the notes are more ${ }_{11}$ distinguishable, as illustrated in Figure 8 [Left-bottom]. However, for clean polyphonic signals, the use of beat po- 12 sitions as prior information for building the transit layer ${ }_{13}$ is too restrictive for having a satisfying decomposition.

When listening to the results on the Mozart excerpt, one ${ }^{14}$ can hear that the residual part obtained with Method ${ }^{15}$ $t r$. is non-negligible compared to the one obtained with ${ }^{16}$ Method (Févotte et al., 2008), although it should be zero. For the Beethoven excerpt, some high frequencies are ${ }^{17}$ captured by the transient basis rather than by the tonal ${ }^{18}$ basis, as it is illustrated Figure 9. Using musical prior ${ }^{19}$ for transients based on the metrical structure thus shows ${ }_{20}$ some potential, as it is adapted to the semantic content ${ }_{21}^{20}$ of the signal, but it should be refined, for instance by using onset positions instead of beat positions. This is also discussed in Section V.E.

\section{Comparison between chromagram versus chords}

We have proposed two methods for building priors for the significance map corresponding to the tonal layer.

1. In the first case (Method Chord), the map is built using information about the tonal content from the estimated chords.

2. In the second case (Method Chroma), the map is built using information about the tonal content directly from the chromagram.

Table V shows that Method Chord outperforms Method Chroma in terms of SNR in the case without noise added 
TABLE III. SNR results (in dB), and chord estimation results (EE: Exact Estimation, E $+N$ : Exact + Neighbor) for various input SNRs and without additional Gaussian noise $(W N)$, for comparison between a semi-automatic (estimated chords) and an automatic (given chords) approach.

\begin{tabular}{|c|c|c|c|c|c|c|c|c|c|}
\hline & $S N R_{i n}$ & \multicolumn{2}{|c|}{$W N$} & \multicolumn{2}{|c|}{0} & \multicolumn{2}{|c|}{10} & \multicolumn{2}{|c|}{20} \\
\hline & Approach & $A$ & $S A$ & $A$ & $S A$ & $A$ & $S A$ & $A$ & $S A$ \\
\hline \multirow{3}{*}{ Gl. } & $E E$ & 14.39 & & 14.39 & & 14.39 & & 14.39 & \\
\hline & $E+N$ & 72.27 & & 72.27 & & 72.27 & & 72.27 & \\
\hline & $S N R_{\text {out }}$ & 71.07 & 71.36 & 14.15 & 14.13 & 21.31 & 21.37 & 28.58 & 28.59 \\
\hline \multirow{3}{*}{ Mi. } & $E E$ & 79.22 & & 79.02 & & 79.22 & & 78.77 & \\
\hline & $E+N$ & 82.00 & & 88.1 & & 82.00 & & 81.54 & \\
\hline & $S N R_{\text {out }}$ & 42.28 & 42.73 & 6.36 & 6.35 & 13.02 & 13.02 & 20.91 & 20.87 \\
\hline \multirow{3}{*}{ Lo. } & $E E$ & 95.29 & & 28.35 & & 60.58 & & 96.27 & \\
\hline & $E+N$ & 100 & & 92.62 & & 100 & & 100 & \\
\hline & $S N R_{\text {out }}$ & 29.12 & 28.31 & 6.44 & 6.42 & 12.44 & 12.44 & 19.24 & 19.21 \\
\hline \multirow{3}{*}{$B e$} & $E E$ & 87.22 & & 86.56 & & 86.77 & & 86.77 & \\
\hline & $E+N$ & 100 & & 100 & & 100 & & 100 & \\
\hline & $S N R_{\text {out }}$ & 55.15 & 54.72 & 7.17 & 7.15 & 13.56 & 13.54 & 21.63 & 21.62 \\
\hline \multirow{3}{*}{ Mo. } & $E E$ & 75.69 & & 70.26 & & 75.25 & & 75.69 & \\
\hline & $E+N$ & 90.26 & & 88.70 & & 90.26 & & 90.26 & \\
\hline & $S N R_{\text {out }}$ & 62.13 & 62.33 & 8.20 & 8.19 & 15.47 & 15.47 & 23.40 & 23.42 \\
\hline
\end{tabular}

TABLE IV. SNRs results (in dB), for various input SNRs and without additional Gaussian noise $(W N)$, for comparison between using musical priors for the transient layer only, case $t r$, and with the baseline approach (Févotte et al., 2008) (F2008).

\begin{tabular}{|l||l|l||l|l||l|l||l|l|}
\hline \multicolumn{1}{|l||}{$S N R_{\text {in }}$} & \multicolumn{2}{c||}{$W N$} & \multicolumn{2}{c||}{0} & \multicolumn{2}{c|}{10} & \multicolumn{2}{c|}{20} \\
\hline Method & $t r$ & F2008 & $t r$ & F2008 & $t r$ & F2008 & $t r$ & F2008 \\
\hline \hline Gl. & 70.01 & 70.22 & 15.54 & 15.74 & 22.14 & 22.45 & 29.16 & 29.22 \\
\hline Mi. & 33.38 & 44.41 & 6.89 & 6.90 & 13.13 & 13.29 & 20.43 & 21.08 \\
\hline Lo. & 27.30 & 29.61 & 6.72 & 6.77 & 12.77 & 12.72 & 19.49 & 19.35 \\
\hline Be. & 39.60 & 54.64 & 7.71 & 7.71 & 13.90 & 14.03 & 21.44 & 21.99 \\
\hline Mo. & 54.47 & 60.96 & 8.98 & 8.97 & 15.87 & 15.94 & 23.59 & 23.88 \\
\hline
\end{tabular}

to the clean signal, and in the case of the monophonic signal. Method Chroma slightly outperforms Method Chord in terms of SNR in the case of polyphonic music and when noise is added to the clean signal. Experiments reveal that the significance maps obtained with Method Chroma are sharper and sparser than those obtained with Method Chord, as illustrated in Figure 10. Moreover, listening tests reveal that the noise induced by the proposed method in the case of Method Chord, is considerably reduced when using Method Chroma, especially when musical priors are used both for the tonal and the transient layers (see for instance the Beethoven excerpt, case $S N R_{\text {in }}=10 \mathrm{~dB}$ ).

However, reconstructed signals using Method Chroma are not systematically more pleasant to listen too. A drawback of Method Chroma is that, because the hier- 44 archy between the 12 pitch classes is less strong than 45 with Method Chord when building the prior for the ${ }_{46}$ tonal layer (because all pitch classes are given a non- 47 zero contribution, whereas in the case of Method Chord, ${ }_{48}$ only 3 pitch classes are considered at each time-instant), 49 the reconstructed signal may select notes that do not 50
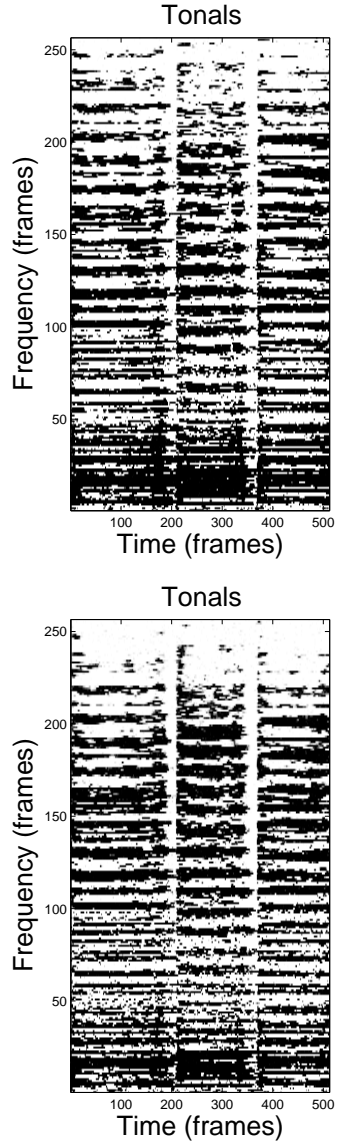

Transients

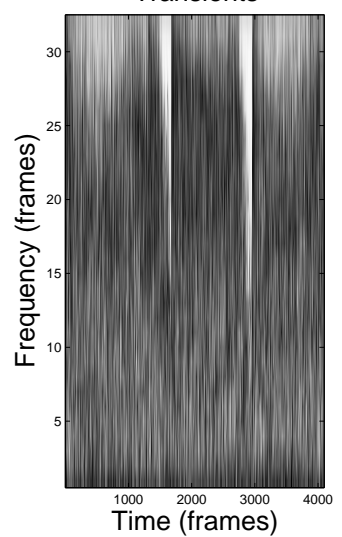

Transients

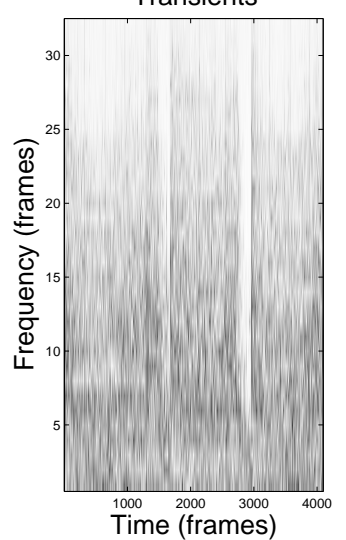

FIG. 9. Significance maps of the tonal and transient bases (MMSE estimates) for the Beethoven excerpt, case $W N$. Top: approach (Févotte et al., 2008); Bottom: proposed approach using musical priors for the transient layer only.

TABLE V. SNRs results (in dB), for various input SNRs and without additional Gaussian noise $(W N)$, for comparison between Method Chord (Cho) and Method Chroma (Chr).

\begin{tabular}{|l||l|l||l|l||l|l||l|l|}
\hline \multicolumn{1}{|l||}{$S N R_{\text {in }}$} & \multicolumn{3}{c||}{$W N$} & \multicolumn{2}{c||}{0} & \multicolumn{2}{c||}{10} & \multicolumn{2}{c|}{20} \\
\hline Method & Cho & Chr & Cho & Chr & Cho & Chr & Cho & Chr \\
\hline \hline Gl. & 71.35 & 69.90 & 14.13 & 13.27 & 21.37 & 20.70 & 28.59 & 27.87 \\
\hline Mi. & 42.73 & 37.09 & 6.35 & 7.04 & 13.02 & 13.35 & 20.87 & 20.89 \\
\hline Lo. & 28.32 & 26.19 & 6.42 & 6.80 & 12.44 & 12.85 & 19.21 & 19.27 \\
\hline Be. & 54.72 & 54.62 & 7.15 & 8.63 & 13.53 & 14.52 & 21.62 & 22.05 \\
\hline Mo. & 62.33 & 60.1 & 8.19 & 9.45 & 15.47 & 16.28 & 23.42 & 23.96 \\
\hline
\end{tabular}

fit the harmonic content, similarly to the one obtained with approach (Févotte et al., 2008). In this case, one may perceive "wrong" notes in the reconstructed signal, especially in high frequencies, as it can be heard, for instance, when listening to the Mozart excerpt, case $S N R_{\text {in }}=10 \mathrm{~dB}$. This blurry phenomenon has especially an impact in the case on clean and monophonic signals. 
TABLE VI. SNR results for comparison between the proposed method that uses musical priors and the approach proposed in (Févotte et al., 2008) (F2008). Case $A$ : musical priors only for the tonal layer (automatic approach). Case $A+t r:$ musical priors for the tonal and the transient layer.

\begin{tabular}{|c|c|c|c|c|c|c|c|c|c|c|c|c|}
\hline$S N R_{\text {in }}$ & \multicolumn{3}{|c|}{$W N$} & \multicolumn{3}{|c|}{0} & \multicolumn{3}{|c|}{10} & \multicolumn{3}{|c|}{20} \\
\hline Method & $A$ & $A+t r$ & F2008 & $A$ & $A+t r$ & F2008 & $A$ & $A+t r$ & F2008 & $A$ & $A+t r$ & F2008 \\
\hline Gl. & 71.35 & 71.49 & 70.22 & 14.13 & 13.86 & 15.74 & 21.37 & 20.98 & 22.45 & 28.59 & 28.24 & 29.22 \\
\hline Mi. & 42.73 & $\overline{32.72}$ & 44.41 & 7.03 & 7.04 & 6.9 & 13.35 & 13.34 & 13.29 & 20.89 & 20.60 & 21.08 \\
\hline Lo. & 28.32 & 27.14 & 29.61 & 6.80 & 6.72 & 6.77 & 12.85 & 12.95 & 12.72 & 19.27 & 19.72 & 19.35 \\
\hline Be. & 54.72 & 35.97 & 54.64 & 8.63 & 8.65 & 7.71 & 14.52 & 14.54 & 14.03 & 22.05 & 21.49 & 21.99 \\
\hline Mo. & 62.33 & 57.07 & 60.96 & 9.45 & 9.37 & 8.97 & 16.28 & 16.12 & 15.94 & 23.96 & 23.73 & 23.88 \\
\hline
\end{tabular}

\section{E. Denoising quality}

Table VI compares results obtained with the proposed 34 method that uses musical priors with the approach pro- 35 posed in (Févotte et al., 2008). As underlined in the pre- 36 vious section, Method Chroma has a negative "blurry" 37 effect on clean and monophonic signals. Ideally, the 38 method should be selected according to the type of test 39 signal. For the sake of legibility, we do not report re- 40 sults with both methods in Table Table VI. Our purpose 41 here is to demonstrate the potential of the proposed mu- 42 sical priors, compared to existing approaches. We thus 43 present the best results, obtained with Method Chroma ${ }_{44}$ in the case of polyphonic music and when noise is added 45 to the clean signal and Method Chord otherwise. Con- 46 cerning the quality of denoising, our model provides re- 47 sults that are comparable to state-of-the-art algorithms 48 in terms of SNR: the difference between our method and 49 method (Févotte et al., 2008) are in general lower than 50 $1 \mathrm{~dB}$. The method proposed in (Févotte et al., 2008) 51 slightly outperforms our method in the case of monophonic music, whereas our method performs slightly better in the case of polyphonic music. Our explanation is 52 that, by relying on chord information, too many notes are considered when building the prior corresponding to ${ }_{53}$ the tonal significance map of the monophonic excerpt. $\mathrm{A}_{54}$ polyphonic/monophonic detection step could be added 55 to improve the proposed model.

Differences between the two approaches may be per- ${ }_{57}$ ceived while listening to the sound files. As said before, ${ }_{58}$ the proposed approach induces some artifacts. In partic- 59 ular, the quality of denoising obtained with the proposed 60 prior for the transient layer is disappointing. Indeed, the ${ }_{61}$ construction of the significance map corresponding to the 62 transient layer leads to some regular "click" sounds that ${ }_{63}$ are superimposed to the signal of interest. As indicated in 64 Table VII and illustrated in Figure 10 and 11, compared 65 to (Févotte et al., 2008), our approach selects much less 66 atoms in the transient layer. Although the idea seems 67 "natural", the use of beat position information for build- 68 ing the prior for the transient layer may be too restrictive 69 for denoising purpose.

When we build the tonal significance map, we select at 71 each time instant all the MDCT bins that correspond to 72 the notes of the estimated chord, regardless of octaves. 73 Because the estimation of the tonal content is rough (we 74 do not have an exact transcription and ignore for instance 75 passing tones), some of the "tubes" that result from this 76 selection in the tonal significance map may correspond to added notes that are not actually in the signal and thus not completely relevant. However, these atoms are musically coherent with the music content (they are coherent with the underlying harmony) and are not as disturbing as if they were atoms randomly selected. Figure 11 shows the significance maps (MMSE estimates) for the Mozart signal, in the case $S N R_{\text {in }}=10 \mathrm{~dB}$. It can be seen that in the case of (Févotte et al., 2008) approach, many atoms that do not fit the tonal content are selected, especially in high frequencies. When listening to the reconstructed signal, one may perceive some notes that seem "wrong". When listening to the signal obtained with the proposed approach, one may also perceive somme added notes, but they are coherent with the harmonic content. This is important when extracting higher-level information from the reconstructed signal. For instance, in the case of key estimation, the algorithm will be less affected by notes that belong to the tonal content than by notes that seem randomly selected.

\section{F. Sparsity}

In the case of polyphonic music, the proposed approach provides a representation that is sparser than the one proposed in (Févotte et al., 2008). Indeed, the number of remaining non-zero coefficients in contrast to the total numbers of atoms of the initial redundant dictionary is usually lower when using musical priors, as well as Renyi entropy, as it can be seen in Table VII. Figures 8, 10 and 11 illustrate the fact that the energy density of the significance map corresponding to the tonal layer is concentrated into thin horizontal lines, and Figures 8, 10 and 12 clearly illustrate that the energy density of the significance map corresponding to the transient layer is is concentrated into thin vertical lines. In the case of monophonic music, figures in Table VII indicate that our representation is less sparse than the one provided in (Févotte et al., 2008). This is because our method induces some artifacts resulting from undesirable selected atoms (see Figure 12, [middle]). The representation may become sparser by increasing the value of $p_{\text {ton }}$ in Eq. (9), as explained in Section V.G.1. Note that, as illustrated in Figure 8, in the case of running the algorithm on a clean signal (case $W N$ ), our approach provides a representation that is sparser than the one obtained with (Févotte et al., 2008), where a small noise is needed to obtain a 
TABLE VII. Percentage of remaining non-zero coefficients selected for the tonal layer (\% ton.) and the transient layer $(\%$ tran.) and value of Renyi entropy for tonal layer (Renyi ton.) and the transient layer (Renyi tran.) (MAP estimates). Case: F2008: approach in (Févotte et al., 2008). Case $A$ : musical priors only for the tonal layer (using estimated chords). Case $A+$ tr: musical priors for the tonal and the transient layer.

\begin{tabular}{|c|c|c|c|c|c|c|c|c|c|c|c|c|c|}
\hline & $S N R_{i n}$ & \multicolumn{3}{|c|}{$W N$} & \multicolumn{3}{|c|}{0} & \multicolumn{3}{|c|}{10} & \multicolumn{3}{|c|}{20} \\
\hline & Method & $A$ & $A+t r$ & F2008 & $A$ & $A+t r$ & F2008 & $A$ & $A+t r$ & F2008 & $A$ & $A+t r$ & F2008 \\
\hline \multirow{4}{*}{$G l$. } & $\%$ ton. & 26.1482 & 19.7510 & 30.3772 & 2.0317 & 1.9966 & 1.5022 & 2.5848 & 2.4879 & 1.5518 & 3.3974 & 3.2333 & 3.3745 \\
\hline & $\%$ tran. & 9.0039 & 33.0078 & 24.6094 & 0 & 8.9844 & 0 & 0 & 6.8359 & 0 & 0 & 5.9570 & 0 \\
\hline & Renyi ton. & 15.0604 & 14.6550 & 15.2776 & 11.3662 & 11.3403 & 10.9392 & 11.7111 & 11.6559 & 10.9812 & 12.1047 & 12.0313 & 12.0875 \\
\hline & Renyi tran. & 15.2006 & 15.3874 & 14.9631 & $N a N$ & 13.5122 & $N a N$ & $N a N$ & 13.1180 & $N a N$ & $N a N$ & 12.9194 & $N a N$ \\
\hline \multirow{4}{*}{ Mi. } & $\%$ ton. & \begin{tabular}{|l|}
23.1979 \\
\end{tabular} & 14.7957 & & & & & & & & & $\overline{9.4261}$ & 27414 \\
\hline & $\%$ tran. & 58.3740 & 32.6172 & 44.8242 & 1.8164 & 16.2109 & 12.3047 & 21.5820 & 19.3359 & 48.9014 & 46.1914 & 23.3398 & 67.8223 \\
\hline & Renyi ton. & 14.8886 & 14.2306 & 16.0161 & 11.9396 & 11.9697 & 4.3331 & 12.7418 & 12.8048 & 15.0260 & 13.5188 & 13.5808 & 15.5774 \\
\hline & Renyi tran. & 16.1770 & 15.3702 & 15.7958 & 13.9016 & 14.3637 & 13.9315 & 14.7733 & 14.6180 & 15.9218 & 15.8713 & 14.8896 & 16.3935 \\
\hline \multirow{4}{*}{ Lo } & \% ton. & 16.6702 & 12.2955 & 45.8679 & 2.6535 & 2.8152 & 11.4395 & 4.8920 & 5.0903 & 20.6650 & 8.7173 & 8.7738 & $\overline{32.9956}$ \\
\hline & $\%$ tran. & 74.0723 & 37.5000 & 27.0020 & 1.2500 & 20.3125 & 0.2051 & 5.7031 & 26.5625 & 19.4824 & 51.9531 & 32.2266 & 24.6582 \\
\hline & Renyi ton. & 13.4112 & 12.9614 & 14.8705 & 10.7474 & 10.8349 & 2.8587 & 11.6348 & 11.6909 & 13.7200 & 12.4686 & 12.4759 & 14.3943 \\
\hline & Renyi tran. & 15.5207 & 14.5736 & 14.0640 & 14.3106 & 13.6891 & 12.6616 & 14.8560 & 14.0761 & 13.5926 & 15.0413 & 14.3550 & 13.932 \\
\hline & $\%$ ton. & 30.2101 & 19.2009 & 44.4839 & 3.1097 & 3.0518 & 8.9005 & 5.6511 & 5.9242 & 18.5188 & 9.5741 & $\overline{10.3973}$ & 32.757 \\
\hline & $\%$ tran. & 57.5195 & 31.2500 & 61.3037 & 2.9297 & 6.8359 & 3.4033 & 23.4375 & 6.7383 & 82.7148 & 80.0781 & 15.8203 & $\overline{99.8779}$ \\
\hline & Renyi ton. & 15.2689 & 14.6063 & 15.8275 & 11.9828 & 11.9540 & 13.5067 & 12.8449 & 12.9090 & 14.5619 & 13.6028 & 13.7216 & 15.383 \\
\hline & Renyi tran. & 16.1560 & 15.3084 & 16.2482 & 11.8713 & 13.1180 & 14.0549 & 14.8926 & 13.0972 & 16.6802 & 16.6664 & 14.3285 & 16.9521 \\
\hline \multirow{4}{*}{ Mo. } & $\%$ ton. & 36.0977 & \begin{tabular}{|l|}
21.1670 \\
\end{tabular} & 42.2409 & 2.9648 & 2.9938 & 8.1978 & 4.6402 & 4.8706 & 12.5305 & 7.4661 & 7.5645 & $\overline{18.7614}$ \\
\hline & $\%$ tran. & 39.4287 & 54.6875 & 33.2764 & 4.1992 & 14.7461 & 14.4043 & 39.3555 & 15.8203 & 47.2656 & 79.9805 & 28.8086 & $\overline{92.5049}$ \\
\hline & Renyi ton. & & & & & 11.9226 & & & & & 13.2364 & 13.2536 & 4.5687 \\
\hline & Renyi tran. & 15.6106 & \begin{tabular}{|l|}
16.1180 \\
\end{tabular} & 15.3662 & 12.4149 & 14.2271 & & 133 & 1422 & & 16.6655 & 15.1933 & 6.841 \\
\hline
\end{tabular}

77 good decomposition.

\section{Indicator variable prior set-up:}

The values $p_{\text {ton }}$ in Eq. (9) and Eq. (10) and $p_{\text {tran }}$ in 27 Eq. (13) have an effect on the above-mentioned artifacts 28 produced by our model in low-input SNRs conditions. 29 For instance, setting $p_{\text {ton }}$ and $p_{\text {tran }}$ to 0.99 instead of 30 0.9 in the case of the Glockenspiel signal allows reducing ${ }_{31}$ the artifacts for $S N R_{\text {in }}=10 \mathrm{~dB}$, as it can be seen in 32 Figure 12. However, our experiments show that indicator 33 variables corresponding to atoms that do not belong to 34 the chord must not be set to 0 . Setting $p_{\text {ton }}$ or $p_{\text {tran }}$ to $1_{35}$ results in reconstructed signals of very "poor" sound, as it 36 can be assessed by listening tests. Output SNRs are also 37 degraded. Setting $p_{\text {ton }}<1$ allows taking into account ${ }_{38}$ imperfections of the chromagram given as input of the 39 hybrid model (temporal imperfections due to windowing,

15 discrepancy between the ideal model and reality, etc.).

\section{2. Impact of tuning:}

Integrating tuning information in the model does not 43 lead to improvement in terms of output SNR values (de- 44 tailed results are not reported here to avoid overfill of the 45 article), but yields to estimated significance maps that 46 are more coherent with our model. Indeed, the "tubes" 47 depend on the tuning and thus, in case of "bad" tuning, 48

\section{Impact of harmonics:}

We did not find any improvement when adding harmonics in our model: the difference between the SNR results obtained with and without considering the harmonics was systematically $\leq 0.3 d B$ (detailed results are not reported here to avoid overfill of the article). This may be explained by the fact that, in the polyphonic case, the contribution of a large part of the first 6 higher harmonics of a note is already taken into account in the significance map by the other notes. For instance, let us consider $\mathrm{C}$ major chord (C-E-G). The $\mathrm{C}$ note generates harmonics $\mathrm{E}$ and $\mathrm{G}$. E and $\mathrm{G}$ are thus both actual played notes and harmonics. Their contribution is already partially taken into account in the significance map in the case of the model "without harmonics" .

\section{CONCLUSION AND FUTURE WORK}

In this article, we have presented a new approach for sparse decomposition of audio signals of music on an overcomplete dictionary made as the union of two MDCT bases. The originality of our model is that, within a Bayesian framework, we introduce musical priors that aim at modeling dependencies between the coefficients of the expansion in a more realistic way than what has been proposed before. The main contribution of the article is 

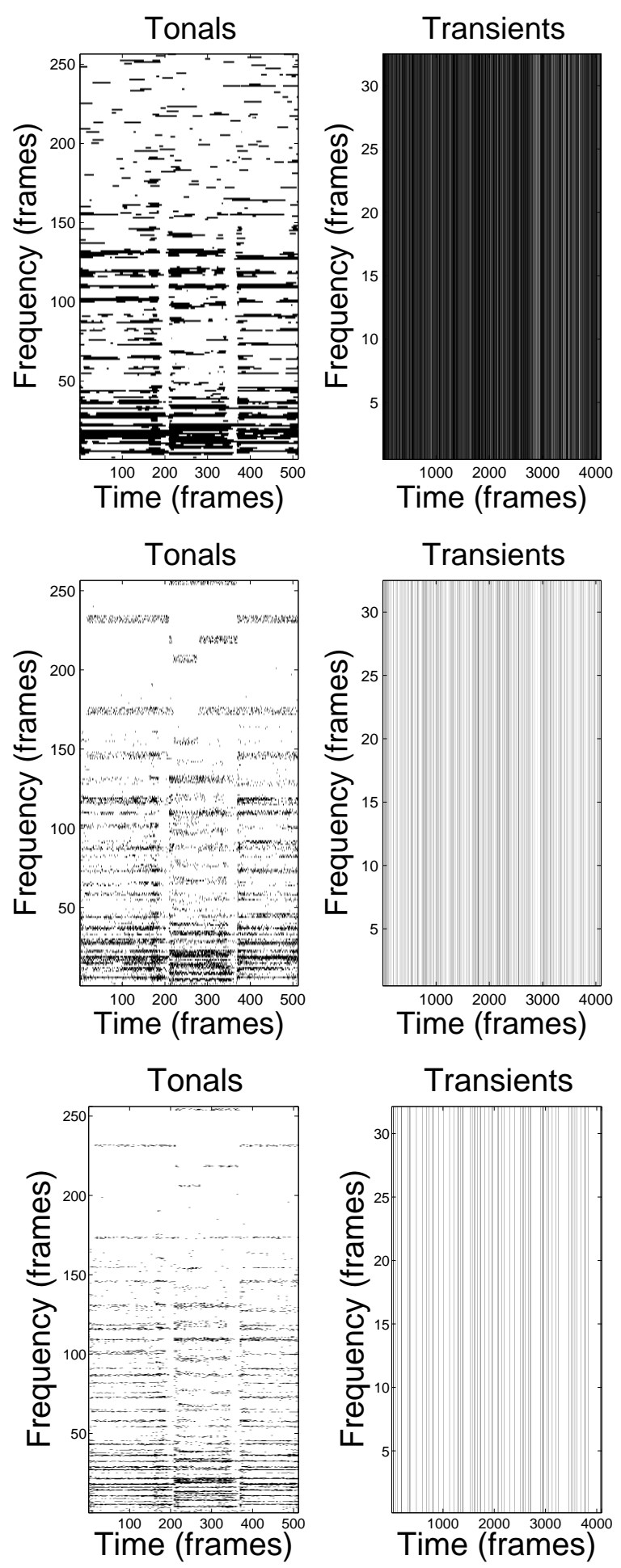

FIG. 10. Significance maps of the tonal and transient bases (MAP estimates) for the Beethoven excerpt, case $S N R_{i n}=$ 10dB. Top: approach (Févotte et al., 2008); middle: proposed approach using musical priors for both layers, Method Chord; bottom: proposed approach using musical priors for both lay- 3 ers, Method Chroma.

to show that the musical prior based on musical knowl- 8 edge performs as well as more sophisticate prior as HMM 9
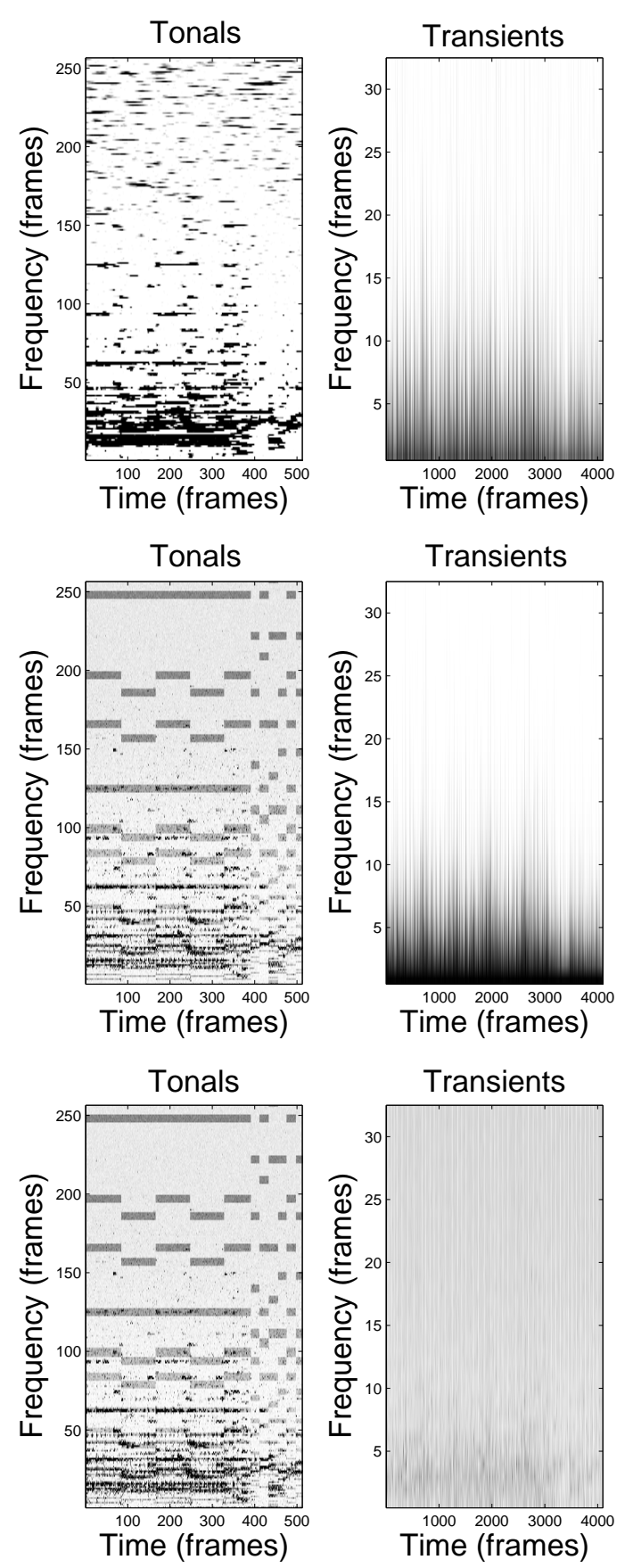

FIG. 11. Significance maps of each basis (MMSE estimates) for the Mozart excerpt, case $S N R_{i n}=10 \mathrm{~dB}$. Top: approach (Févotte et al., 2008); middle: proposed approach using a musical prior for the tonal layer; bottom: proposed approach using musical priors for both the tonal and transient layers.

and appears to be more "natural". The significance maps corresponding to the tonal and transient layers are coherent with the intrinsic content of music audio.

We have provided numerical results and a number of case study examples that assess that our model is adequate to fairly represent audio signals of music. The denoising task has been used as a "proof of concept" of the 

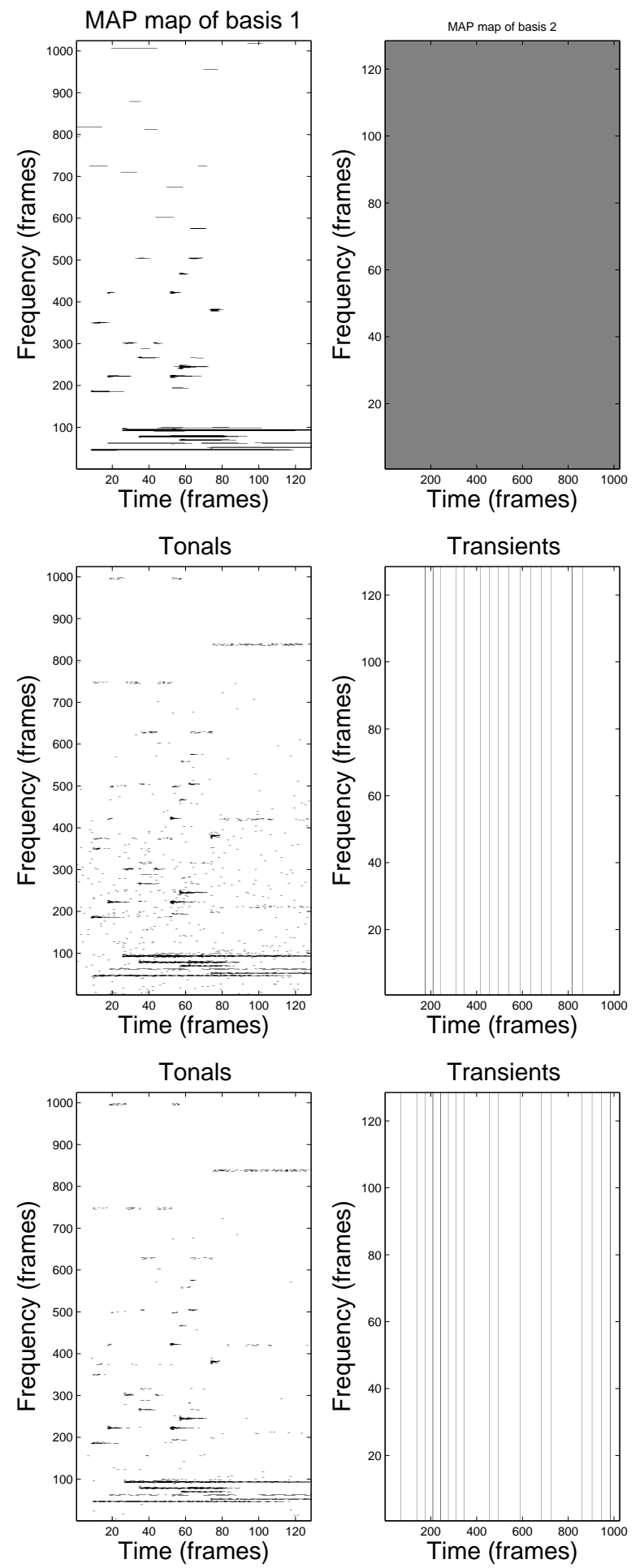

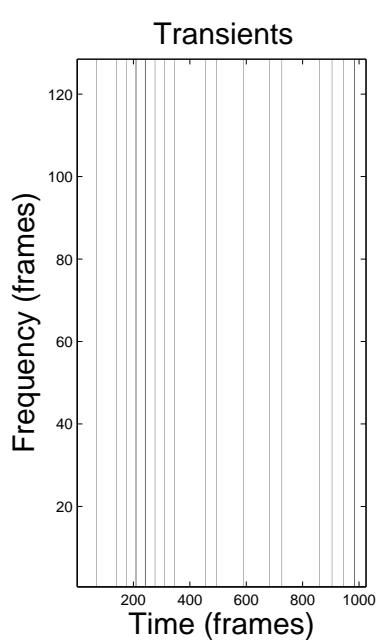

propose provide results whose quality in terms of SNR results outperforms or, at least, corresponds to state-of-theart approaches. Moreover, the content of reconstructed signal is more coherent with the underlying harmony and metrical structure, and thus musically meaningful.

A well-structured representation may be very useful to provide access to higher level information about the audio signal, whereas relevant music content information should help providing a "good" representation. Future work will concentrate on fully integrating in the model chord and beat estimation in an interactive fashion. For instance the chromagram could be updated with the other parameters during MCMC inference in order to possibly improve the chord estimation.

The priors we propose have a great potential of improvement in the future (for example, by using a time segmentation, a larger chord lexicon, using onsets combined with beat positions etc.). The model could also be extended so that dependencies between layers are taken into account. For this, musical information such as the fact that chord changes usually occur on beat subdivisions (which are related to transient locations) could be used. This should help reducing musical noise and artifacts.

As far as we know, the introduction of musical priors in hybrid models for spare decomposition is novel. The use of mid-level representation of audio - such as the chromagram, as proposed in this paper - or scores, if available, could be extended to many applications such as denoising, source separation, compression, coding and many others. Usually, only physical and mathematical criteria are taken into account. We believe that the use of musical content information opens new interesting perspectives.

\section{Acknowledgment}

The authors would like to thank C. Févotte and all the authors of (Févotte et al., 2008) for providing their source code.

\section{APPENDIX A: POSTERIOR DISTRIBUTIONS USED IN THE GIBBS SAMPLER}

\section{Indicator variables}

\section{a. Tonal layer}

$$
\begin{aligned}
& p\left(\gamma_{\text {ton }, n}=0 \mid \sigma_{\text {ton }, n}, \sigma, x_{\text {ton } \mid \text { tran }}\right)=\frac{1}{1+\tau_{\text {ton }, n}} \\
& p\left(\gamma_{\text {ton }, n}=1 \mid \sigma_{\text {ton }, n}, \sigma, x_{\text {ton } \mid \text { tran }}\right)=\frac{\tau_{\text {ton }, n}}{1+\tau_{\text {ton }, n}}
\end{aligned}
$$

10 newly introduced musical priors. Concerning the qual-

11 ity of denoising, all the configurations of the model we 41 with 


$$
\begin{aligned}
\tau_{t o n, n}= & \sqrt{\frac{\sigma^{2}}{\sigma^{2}+\sigma_{t o n, n}}} \exp \left(\frac{x_{t o n \mid \operatorname{tran}}^{2} \sigma_{t o n, n}}{2 \sigma^{2}\left(\sigma^{2}+\sigma_{\text {ton }, n}\right)}\right) \\
& \times p\left(\frac{\gamma_{t o n, n}=1 \mid \gamma_{t o n,-n}}{\gamma_{t o n, n}=0 \mid \gamma_{t o n,-n}}\right) .
\end{aligned}
$$

$$
\begin{array}{r}
p\left(\sigma_{\text {ton }, n}^{2} \mid \gamma_{\text {ton }, n}, f_{\text {ton }, n}\right)=\left(1-\gamma_{\text {ton }, n}\right) \mathcal{I} \mathcal{G}\left(\sigma_{\text {ton }, n} \mid 1, f_{\text {ton }, n}\right) \\
+\gamma_{\text {ton }, n} \mathcal{I} \mathcal{G}\left(\sigma_{\text {ton }, n} \mid 3 / 2, \frac{\alpha_{\text {ton }, n}^{2}}{2}+f_{\text {ton }, n}\right)
\end{array}
$$

$p\left(\sigma_{\text {tran }, m}^{2} \mid \gamma_{\text {tran }, m}, f_{\text {tran }, m}\right)=\left(1-\gamma_{\text {tran }, m}\right) \mathcal{I} \mathcal{G}\left(\sigma_{\text {tran }, m} \mid 1, f_{\text {tran }, m}\right)$

$$
+\gamma_{\text {tran }, m} \mathcal{I} \mathcal{G}\left(\sigma_{\text {tran }, m} \mid 3 / 2, \frac{\alpha_{\text {tran }, m}^{2}}{2}+f_{\text {tran }, m}\right)
$$

$$
\begin{aligned}
& p\left(\gamma_{\text {tran }, m}=0 \mid \sigma_{\text {tran }, m}, \sigma, x_{\text {tran } \mid \text { ton }}\right)=\frac{1}{1+\tau_{\text {tran }, m}} \\
& p\left(\gamma_{\text {tran }, m}=1 \mid \sigma_{\text {tran }, m}, \sigma, x_{\text {tran } \mid \text { ton }}\right)=\frac{\tau_{\text {tran }, m}}{1+\tau_{\text {tran }, m}}
\end{aligned}
$$

46 with

$$
\begin{aligned}
\tau_{\text {tran }, m}= & \sqrt{\frac{\sigma^{2}}{\sigma^{2}+\sigma_{\text {tran }, m}}} \exp \left(\frac{x_{\text {tran } \mid \operatorname{ton}}^{2} \sigma_{\text {tran }, m}}{2 \sigma^{2}\left(\sigma^{2}+\sigma_{\text {tran }, m}\right)}\right) \\
& \times p\left(\frac{\gamma_{\text {tran }, m}=1 \mid \gamma_{\text {tran },-m}}{\gamma_{\text {tran }, m}=0 \mid \gamma_{\text {tran },-m}}\right) .
\end{aligned}
$$$$
p\left(\lambda_{\text {ton }} \mid \sigma_{\text {ton }}\right)=\gamma\left(\lambda_{\text {ton }} \mid N, \sum_{n} \frac{1}{1+\frac{q-1}{\ell_{\text {ton }} / 3} \sigma_{\text {ton }, n}}\right)
$$$$
p\left(\lambda_{\text {tran }} \mid \sigma_{\text {tran }}\right)=\gamma\left(\lambda_{\text {tran }} \mid N, \sum_{m} \frac{1}{1+\frac{q-1}{\ell_{\text {tran }} / 3} \sigma_{\text {tran }, m}}\right)
$$

\section{c. Variance of the noise}

$p\left(\sigma^{2} \mid x_{\text {ton }}, x_{\text {tran }}, x\right)=\mathcal{I} \mathcal{G}\left(\sigma^{2} \mid \frac{N}{2}, \frac{\|x-V \alpha-U \beta\|^{2}}{2}\right)$

49 2. Coefficients

$$
\begin{aligned}
& p\left(\alpha_{n} \mid \gamma_{t o n, n}, \sigma_{\text {ton }, n}, \sigma, x_{\text {ton } \mid \text { tran }}\right)=\left(1-\gamma_{\text {ton }, n}\right) \delta_{0}\left(\alpha_{n}\right) \\
& +\gamma_{\text {ton }, n} \mathcal{N}\left(\alpha_{n} \mid \frac{u_{n}^{T} r \sigma_{\text {ton }, n}^{2}}{\sigma_{\text {ton }, n}^{2}+\sigma^{2}}, \frac{\sigma^{2} \sigma_{\text {ton }, n}^{2}}{\sigma^{2}+\sigma_{\text {ton }, n}^{2}}\right)_{62}^{{ }_{61}^{50}} \\
& +\gamma_{\text {tran }, m} \mathcal{N}\left(\beta_{m} \mid \frac{v_{m}^{T} r \sigma_{\text {tran }, m}^{2}}{\sigma_{\text {tran }, m}^{2}+\sigma^{2}}, \frac{\sigma^{2} \sigma_{\text {tran }, m}^{2}{ }^{72}}{\sigma^{2}+\sigma_{\text {tran }, m}^{2}}\right)
\end{aligned}
$$

(A6) ${ }_{75}^{74}$
1. See e.g. https://sites.google.com/site/nips10sparsews/.

2. Here $\gamma$ is used to refer indifferently to $\gamma_{\text {ton,n }}$ or $\gamma_{\text {tran }, m}$.

3. $a(\bmod b)$ denotes the mathematical operator modulo, the remainder when $a$ is divided by $b$.

4. In general, the harmonics of harmonic music sounds do not have frequencies that are exact multiples of its fundamental frequency, but are nearly harmonically related.

5. We limit the number of considered harmonics to 6 because many of the higher harmonics, which are theoretically whole number multiples of the fundamental frequency, are far from any note of the Western chromatic scale. This is especially true for the 7 th and the 11th harmonics.

6. As stressed in (Févotte et al., 2008), colored or non-Gaussian noise could also be considered as well and embedded into the same framework, but this would lead to an increase in the computational efficiency of the algorithm because of some matrix inversion, which is out of the scope of this paper.

7. Neighboring chords considered here are harmonically close triads: parallel Major/ minor (EM being confused with Em), relative (Am being confused with $\mathrm{CM}$ ), dominant (CM being confused with GM) or subdominant (CM being confused with FM). 
Baraniuk, R., Flandrin, P., Janssen, A., and Michel, O. 60 (2001). "Measuring Time-Frequency Information Content 61 Using the Rényi Entropies", Proceedings of the IEEE Trans- 62 actions on Information Theory 47, 1391-1409.

Bello, J. and Pickens, J. (2005). "A robust mid-level represen- 64 tation for harmonic content in music signal", in Proceedings of 65 the International Symposium on Music Information Retrieval 66 (ISMIR) (London, UK).

Benaroya, L., Bimbot, F., and Gribonval, R. (2006). "Audio 68 source separation with a single sensor", IEEE Transactions 69 on Audio, Speech, and Language Processing 14, 191-199. 70 Blumensath, T. and Davies, M. (2004). "Unsupervised learn- 71 ing of sparse and shift-invariant decompositions of polyphonic 72 music", in Proceedings of the IEEE International Conference 73 on Acoustics, Speech, and Signal Processings (ICASSP), vol- 74 ume 5, 497-500 (Montreal, Canada).

Brown, J. (1991). "Calculation of a constant Q spectral trans- 76 form", Journal of the Acoustical Society of America 89, 425- 77 434.

Casella, G. and George, E. (1992). "Explaining the Gibbs 79 sampler", The American Statistician 46, 167-174.

Chen, S., David L. Donoho, D., and Saunders, M. (1998). 81 "Atomic decomposition by basis pursuit", SIAM Journal on 82 Scientific Computing 20, 33-61.

Crouse, M., Nowak, R., and Baraniuk, R. (1998). "Wavelet- 84 based statistical signal processing using hidden markov mod- 85 els", IEEE Transactions on Signal Processing 46, 886-902. $\quad 86$ Daudet, L. (2004). "Sparse and structured decompositions of 87 audio signals in overcomplete spaces", in Proceedings of the 88 International Conference on Digital Audio Effects (DAFx), 89 22-26 (Naples, Italy).

Daudet, L. (2006a). "A review on techniques for the ex-91 traction of transients in musical signals", in Computer $M u-92$ sic Modeling and Retrieval, volume 3902 of Lecture Notes in 93 Computer Science, 219-232 (Springer-Verlag Berlin Heidel- 94 berg).

Daudet, L. (2006b). "Sparse and structured decompositions 96 of signals with the molecular matching pursuit", IEEE Trans- 97 actions on Audio, Speech, and Language Processing 14, 1808- 98 1816.

Daudet, L. (2010). "Audio sparse decompositions in paral-100 lel, Let the greed be shared!", IEEE Transactions on Signal 101 Processing 27, 90-96.

Daudet, L., Molla, S., and Torrésani, B. (2004). "Towards103 a hybrid audio coder", in Proceedings of the International 104 Conference Wavelet Analysis Its Applications (WAA), 13-24105 (Chongqing, Chine).

Daudet, L. and Torrésani, B. (2002). "Hybrid representations107 for audiophonic signal encoding", Signal Processing Journal 108 82, 1595-1617.

Daudet, L. and Torrésani, B. (2006). Signal Processing Meth-110 ods for Music Transcription, chapter "Sparse Adaptive Rep-111 resentations for Musical Signals", 65-98 (Springer US).

Davies, M. and Daudet, L. (2006). "Sparse audio representa-113 tions using the MCLT", Signal Processing Journal 86, 457-114 470.

Davies, M. and Plumbley, M. (2007). "Context-dependent116 beat tracking of musical audio". IEEE Transactions on Audio,117 Speech, and Language Processing 15, 1009-1020.

Dixon, S. (2007). "Evaluation of audio beat tracking system119 beatroot", Journal of New Music Research 36, 39-51.

Emiya, V., Vincent, E., Harlander, N., and Hohmann, V.121 (2010). "Subjective and objective quality assessment of audio122 source separation", Rapport de recherche RR-7297, INRIA. 123 Févotte, C., Daudet, L., Godsill, S., and Torresani, B.124 (2006). "Sparse regression with structured priors: Applica-125 tion to audio denoising", in Proceedings of the IEEE International Conference on Acoustics, Speech, and Signal Processings (ICASSP), volume 3, III (Toulouse, France).

Févotte, C. and Godsill, S. (2006). "A Bayesian approach for blind separation of sparse sources", IEEE Transactions on Audio, Speech, and Language Processing 14, 2174-2188.

Févotte, C. and Godsill, S. (2006). "Sparse linear regression in unions of bases via Bayesian variable selection", IEEE Signal Processing Letters 13, 441-444.

Févotte, C., Torrésani, B., Daudet, L., and Godsill, S. (2008). "Sparse Linear Regression With Structured Priors and Application to Denoising of Musical Audio", IEEE Transactions on Audio, Speech, and Language Processing 16, 174-185.

Figueiredo, M. (2003). "Adaptive Sparseness for Supervised Learning", IEEE Transactions on Pattern Analysis and Machine Intelligence 25, 1150-1159.

Fujishima, T. (1999). "Real-time chord recognition of musical sound: a system using common lisp music", in Proceedings of the International Computer Music Conference (ICMC), 464467 (Bejing, China).

Geman, S. and Geman, D. (1984). "Stochastic relaxation, Gibbs distributions, and the Bayesian restoration of images", IEEE Transactions on Pattern Analysis and Machine Intelligence 6, 721-741.

George, E. and McCulloch, R. (1997). "Approaches for Bayesian variable selection", Statistica Sinica 7, 339-373. Geweke, J. (1996). "Variable Selection and Model Comparison in Regression", Bayesian Statistics 5, 609-620.

Hamdy, K., Ali, M., and Tewfi, A. (1996). "Low bit rate high quality audio coding with combined harmonic and wavelet representations", in Proceedings of the IEEE International Conference on Acoustics, Speech, and Signal Processings (ICASSP), volume 2, 1045-1048 (Atlanta, Georgia, USA). Harte, C. and Sandler, M. (2005). "Automatic chord identification using a quantised chromagram", in Proceedings of the Convention Audio Engineering Society (AES) (Barcelona, Spain).

Jaillet, F. and Torrésani, B. (2004). "Time-frequency jigsaw puzzle- Adaptive multiwindow and multilayered gabor expansions", Technical report, LATP and Université de Provence. Klapuri, A., Eronen, A., and Astola, J. (2006). "Analysis of the meter of acoustic musical signals", IEEE Transactions on Audio, Speech, and Language Processing 14, 342-355.

Kowalski, M. (2009). "Sparse regression using mixed norms", Applied and Computational Harmonic Analysis 27, 303-324. Kowalski, M. and Torrésani, B. (2008). "Random models for sparse signals expansion on unions of bases with application to audio signals", IEEE Transactions on Signal Processing 56, 3468-3481.

Liuni, M., Roebel, A., Romito, M., and Rodet, X. (2011). "An entropy Based method for Local time-adaptation of the spectrogram", in Computer Music Modeling and Retrieval, Lecture Notes in Computer Science, 60-75 (Springer-Verlag Berlin Heidelberg).

Low, F. (1985). "Complete sets of wave packets", C. DeTar (editor), A Passion for Physics - Essay in Honor of Geoffrey Chew, World Scientific 17-22.

Mallat, S. (1998). A Wavelet Tour of Signal Processing, 3rd edition (Academic Press, San Diego, CA, USA), 832 p.

Mallat, S. and Zhang, Z. (1993). "Matching Pursuit With Time-Frequency Dictionaries", IEEE Transactions on Signal Processing 41, 3397-3415.

Malvar, H. (1990). "Lapped transforms for efficient transform/subband coding", IEEE Transactions on Acoustics, Speech, and Signal Processing 38, 969-978.

Molla, S. and Torésani, B. (2004). "Determining local tran- 
sientness of audio signals", IEEE Signal Processing Letters454 11, 625-628.

Molla, S. and Torésani, B. (2005). "An hybrid audio scheme 456 using hidden Markov models of waveforms", Applied and 457 Computational Harmonic Analysis 18, 137-166.

Noland, K. and M., S. (2006). "Key estimation using a hid 4459 den Markov model", in Proceedings of the International Sym ${ }_{\sharp 460}$ posium on Music Information Retrieval (ISMIR) (Victoria,461 BC, Canada).

Papadopoulos, H. and Kowalski, M. (2011). "Sparse Signal463 Decomposition on Hybrid Dictionaries Using Musical Priors" $\$ 464$ in Proceedings of the International Symposium on Music In ${ }_{ \pm 465}$ formation Retrieval (ISMIR) (Miami, USA).

Papadopoulos, H. and Peeters, G. (2007). "Large-Scale Study467 of Chord Estimation Algorithms Based on Chroma Represen $₫ 468$ tation and HMM", in Proceedings of the International Work ${ }_{4469}$ shop on Content-Based Multimedia Indexing (CBMI), 53-60470 (Bordeaux, France).

Papadopoulos, H. and Peeters, G. (2011). "Joint estimation of chords and downbeats", IEEE Transactions on Audio, Speech, and Language Processing 19, 138-152.

Pati, Y., Rezaiifar, R., and Krishnaprasad, P. (1993). "Orthogonal matching pursuit: Recursive function approximation with applications to wavelet decomposition", in Proceedings of the 27th Annual Asilomar Conference on Signals, Systems, and Computers, 40-44 (Pacific Grove, CA , USA).

Peeters, G. (2006). "Musical key estimation of audio signal based on HMM modeling of chroma vectors", in Proceedings of the International Conference on Digital Audio Effects (DAFx), 127-131 (Montreal, Canada).

Peeters, G. and Papadopoulos, H. (2011). "Simultaneous beat and downbeat-tracking using a probabilistic framework: theory and large-scale evaluation", IEEE Transactions on Audio, Speech, and Language Processing 19, 1754-1769.

Plumbley, M., Blumensath, T., Daudet, L., Gribonval, R., and Davies, M. (2010). "Sparse Representations in Audio and Music: from Coding to Source Separation", Proceedings of the IEEE 98, 995-1005.

Ravelli, E., Richard, G., and Daudet, L. (2008). "Union of MDCT Bases for Audio Coding", IEEE Transactions on Audio, Speech, and Language Processing 16, 1361-1372. Ravelli, E., Richard, G., and Daudet, L. (2010). "Audio signal representations for indexing in the transform domain", IEEE Transactions on Audio, Speech, and Language Processing 18, 434-446.

Rényi, A. (1961). "On measures of entropy and information", in Proceedings of the fourth Berkeley Symposium on Mathematics of Statistics and Probability, volume 41, 547-561 (University of Calif. Press).

Rohdenburg, T., Hohmann, V., , and Kollmeier, B. (2005). "Objective perceptual quality measures for the evaluation of noise reduction schemes", in Proceedings of the 9th International Workshop on Acoustic Echo and Noise Control (IWAENC), 169-172 (Eindhoven, The Netherlands).

Scheirer, E. (1998). "Tempo and beat analysis of acoustic musical signals", Journal of the Acoustical Society of America 103, 588-601.

Sheh, A. and Ellis, D. (2003). "Chord segmentation and recognition using EM-trained HMM", in Proceedings of the International Symposium on Music Information Retrieval (ISMIR) (Baltimore, MD, USA).

Tibshirani, R. (1996). "Regression shrinkage and selection via the lasso", Journal of the Royal Statistical Society Serie B (Statistical Methodology) 58, 267-288.

Verma, T. and Meng, T. (2000). "Extending Spectral Modeling Synthesis with Transient Modeling Synthesis", Computer
Music Journal 24, 47-59.

Vincent, E., Jafari, M., and Plumbley, M. D. (2006). "Preliminary guidelines for subjective evaluation of audio source separation algorithms", in Proceedings of the UK ICA Research Network Workshop, 93-96 (Southampton, Royaume-Uni). Wakefield, G. (1999). "Mathematical representation of joint time-chroma distribution", in Proceedings of the SPIE Conference on Advanced Signal Processing Algorithms, Architecture and Implementation (ASPAAI), 637-645 (Denver, Colorado, USA).

Wolfe, P., Godsill, S., and Ng, W.-J. (2004). "Bayesian variable selection and regularization for time-frequency surface estimation", Journal of the Royal Statistical Society Serie B (Statistical Methodology) 66, 575-589.

Yeh, C., Roebel, A., and Rodet, X. (2010). "Multiple Fundamental Frequency Estimation and Polyphony Inference of Polyphonic Music Signals", IEEE Transactions on Audio, Speech, and Language Processing 18, 1116-1126. 